\title{
Spatially Resolved Decoherence of Donor Spins in Silicon Strained by a Metallic Electrode
}

\author{
V. Ranjan $\odot,{ }^{1}$ B. Albanese, ${ }^{1}$ E. Albertinale, ${ }^{1}$ E. Billaud, ${ }^{1}$ D. Flanigan $\odot,{ }^{1}$ J. J. Pla, ${ }^{2}$ T. Schenkel, ${ }^{3}$ D. Vion, ${ }^{1}$ \\ D. Esteve $\odot,{ }^{1}$ E. Flurin, ${ }^{1}$ J. J. L. Morton, ${ }^{4}$ Y. M. Niquet, ${ }^{5}$ and P. Bertet ${ }^{1, *}$ \\ ${ }^{1}$ Université Paris-Saclay, CEA, CNRS, SPEC, 91191 Gif-sur-Yvette Cedex, France \\ ${ }^{2}$ School of Electrical Engineering and Telecommunications, University of New South Wales, \\ Anzac Parade, Sydney, New South Wales 2052, Australia \\ ${ }^{3}$ Accelerator Technology and Applied Physics Division, Lawrence Berkeley National Laboratory, \\ Berkeley, California 94720, USA \\ ${ }^{4}$ London Centre for Nanotechnology, University College London, London WC1H OAH, United Kingdom \\ ${ }^{5}$ Université Grenoble Alpes, CEA, IRIG-MEM-L_SIM, 38000 Grenoble, France
}

(Received 11 January 2021; accepted 15 June 2021; published 16 August 2021)

\begin{abstract}
Electron spins are amongst the most coherent solid-state systems known. However, to be used in devices for quantum sensing and information processing applications, they must typically be placed near interfaces. Understanding and mitigating the impacts of such interfaces on the coherence and spectral properties of electron spins is critical to realizing such applications, but it is also challenging: Inferring such data from single-spin studies requires many measurements to obtain meaningful results, while ensemble measurements typically give averaged results that hide critical information. Here, we report a comprehensive study of the coherence of near-surface bismuth donor spins in 28 -silicon at millikelvin temperatures. In particular, we use strain-induced frequency shifts caused by a metallic electrode to infer spatial maps of spin coherence as a function of position relative to the electrode. By measuring magnetic-field-insensitive clock transitions, we separate magnetic noise caused by surface spins from charge noise. Our results include quantitative models of the strain-split spin resonance spectra and extraction of paramagnetic impurity concentrations at the silicon surface. The interplay of these decoherence mechanisms for such near-surface electron spins is critical for their application in quantum technologies, while the combination of the strain splitting and clock transition extends the coherence lifetimes by up to 2 orders of magnitude, reaching up to $300 \mathrm{~ms}$ at a mean depth of only $100 \mathrm{~nm}$. The technique we introduce here to spatially map coherence in near-surface ensembles is directly applicable to other spin systems of active interest, such as defects in diamond, silicon carbide, and rare earth ions in optical crystals.
\end{abstract}

DOI: $10.1103 /$ PhysRevX.11.031036

\section{INTRODUCTION}

The electron and nuclear spins of donors in silicon are promising qubit candidates for solid-state quantum computing [1-8] because of their exceptionally long coherence times when the silicon substrate is isotopically enriched in the nuclear-spin-free isotope ${ }^{28} \mathrm{Si}$, reaching seconds for the donor electron spin $[9,10]$ and minutes for its nuclear spin [5]. Donors in silicon are therefore also suitable for implementing long-lived and multimode microwave quantum memories for superconducting qubits [11-13].

\footnotetext{
*patrice.bertet@cea.fr
}

Published by the American Physical Society under the terms of the Creative Commons Attribution 4.0 International license. Further distribution of this work must maintain attribution to the author(s) and the published article's title, journal citation, and DOI.
Subject Areas: Condensed Matter Physics, Quantum Physics
For quantum information processing applications, the donors need to be close to the silicon/silicon-oxide interface and also to the metallic electrodes used to apply the electromagnetic signals enabling quantum state manipulation and readout. However, interfaces have large defect densities, consisting of dangling bonds, surface adsorbents, and tunneling two-level systems (TLS). Moreover, the electrodes are often made with materials that may have thermal expansion coefficients different from the substrate, resulting in spatially dependent mechanical strain. For future donor-based quantum devices, it is essential to understand how the spectrum and coherence time of donors in silicon are modified by the proximity of interfaces with the surface oxide [14-16] and with the electrodes. While single-donor devices provide detailed information on the behavior of individual dopants $[5,17]$, they would require a prohibitively large number of measurements for extracting spatially resolved properties on a larger scale. Here, we 
achieve this goal with an ensemble of donors in silicon in the vicinity of a micron-wide aluminum electrode. Using strain-induced frequency shifts combined with extensive coherence time measurements, we infer magnetic and charge noise as a function of depth and position relative to the electrode. In addition to insights into the decoherence mechanisms at play for near-surface donors in silicon, our results are of broader significance in two distinct ways: First, the techniques of spatial mapping can be readily applied to other spins that show coupling to strain, not just in silicon (such as acceptors [18]) but also systems such as NV (nitrogen vacancy) centers in diamond [19] or rare earth ions [20]. Second, spins with an adjustable sensitivity to magnetic-field noise, such as the Bi donors used here, act as a versatile probe to distinguish magnetic and charge noise near an interface, which can be critical for the performance of, e.g., superconducting qubits [21].

The measurements are made possible by the properties of bismuth donors in silicon, a particularly interesting system to probe the physical mechanisms at play near interfaces. Because of the strong hyperfine interaction of the donor electron spin $S=1 / 2$ with the $I=9 / 2$ nuclear spin of the bismuth atom, bismuth donors are sensitive to strain [22] and electric fields, and have transitions with widely varying effective gyromagnetic ratios, which allows us to distinguish the various sources of noise by comparing coherence times on different transitions.

Our device consists of an ensemble of shallow-implanted bismuth donors in a silicon substrate isotopically enriched in ${ }^{28} \mathrm{Si}$, magnetically coupled to an aluminum wire deposited on top of the substrate. The wire is the inductor in a superconducting $L C$ resonator used for quantum-limited electron paramagnetic resonance (EPR) spectroscopy at millikelvin temperatures [23-25] (Secs. II and III). The bismuth donor spin spectrum of a similar device was shown to be governed by the strain imparted by the differential thermal contraction of the aluminum wire with respect to the underlying silicon substrate $[26,27]$. Here, we confirm these results by showing that such a model also quantitatively predicts the line shape when varying the wire width (Sec. IV). Furthermore, we use the phenomenon of instantaneous diffusion to demonstrate that strain is locally inhomogeneous (Sec. V). We then study the Hahnecho coherence time $T_{2}$ for various spin transitions, wire geometries, and strain values. We observe a strong dependence of $T_{2}$ on strain. Because in our device geometry the strained donors are also closer to the surface, this provides evidence that $T_{2}$ is limited by magnetic noise originating from the device interfaces, in agreement with previous work $[14,16]$. Taking advantage of the strain gradient around the wire, our measurements provide some amount of spatial resolution, indicating a paramagnetic defect density of $4 \times 10^{12} \mathrm{~cm}^{-2}$ away from the wire, and $10^{12} \mathrm{~cm}^{-2}$ below the wire (Sec. VI). We finally measure $T_{2}$ on a magnetic-noise-insensitive clock transition, finding, again, a strong dependence on strain, with the largest measured value being $T_{2}=300 \mathrm{~ms}$. We argue that this $T_{2}$ is limited by charge noise at the silicon/siliconoxide interface (Sec. VII). We support our findings with complementary measurements of both the magnetic and charge noise dependence on pulse sequence (via dynamical decoupling experiments), temperature, and artificially introduced microwave noise (Sec. VIII).

\section{BISMUTH DOPANTS IN SILICON}

We briefly summarize the properties of bismuth donor spins in silicon that are relevant to this work. More details can be found in Refs. [28,29] and the Appendix A.

As a group- $\mathrm{V}$ element, bismuth is an electron donor when inserted in the silicon lattice where it forms four covalent bonds. At low temperature, the extra electron $(S=1 / 2)$ can be trapped around the $\mathrm{Bi}^{+}$ion (with nuclear spin $I=9 / 2$ ) by the Coulomb interaction, forming a coupled electron-nuclear spin system [28]. Group-V donors have a symmetric $s$-wave electronic wave function in the ground state, similar to a hydrogen atom, albeit with a Bohr radius spanning several lattice constants [Fig. 1(a)]. The Hamiltonian of a donor spin system under a static magnetic field $B_{0}$ is given by

$$
H / \hbar=A \mathbf{S} \cdot \mathbf{I}+\left(\gamma_{e} \mathbf{S}+\gamma_{n} \mathbf{I}\right) \cdot \mathbf{B}_{\mathbf{0}},
$$

where $\gamma_{e} / 2 \pi=28 \mathrm{MHz} / \mathrm{mT}$ and $\gamma_{n} / 2 \pi=7 \mathrm{kHz} / \mathrm{mT}$ are the electronic and nuclear gyromagnetic ratios, and $A / 2 \pi=$ $1.475 \mathrm{GHz}$ is the hyperfine coupling constant between electron and nuclear spins. One specificity of bismuth donor spins compared to other group- $\mathrm{V}$ donors such as phosphorus is the large value of $A$. This value makes it possible to explore the low-field limit $\gamma_{e}\left|B_{0}\right| \ll A$ (corresponding to $B_{0}<10-20 \mathrm{mT}$ ), in which the energy eigenstates' wave functions are hybridized electronuclear spin wave functions dominated by the hyperfine interaction. All the results presented here are in this low-field limit.

Under Hamiltonian Eq. (1), the total angular-momentum projection $m$ on the $z$ direction, along which $B_{0}$ is applied, is a good quantum number, and the total angular momentum (of the operator $\mathbf{F}=\mathbf{S}+\mathbf{I}$ ) is an approximate one in the low-field limit. The energy levels can be determined numerically or analytically [29] and are shown in Fig. 1(c). They can be approximated by states $|F, m\rangle$ and are distributed into a low-energy $(F=4)$ manifold of nine states and a high-energy $(F=5)$ manifold of 11 states separated by a zero-field splitting (ZFS) of $5 A / 2 \pi=7.375 \mathrm{GHz}$. The ZFS makes it particularly suitable for EPR spectroscopy using superconducting resonators since it can be performed even at low magnetic fields if the resonator frequency is close to the ZFS.

EPR transitions driven resonantly by a transverse ac magnetic field $B_{1}$ are allowed between levels satisfying $|\Delta m|=1$. In Fig. 1(d), we show the 18 allowed EPR 
(a)

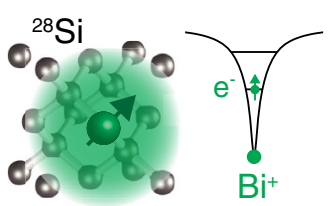

(b)

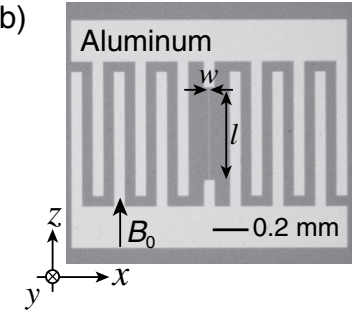

(g)

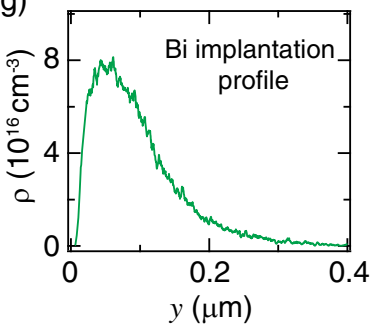

(c)

(d)

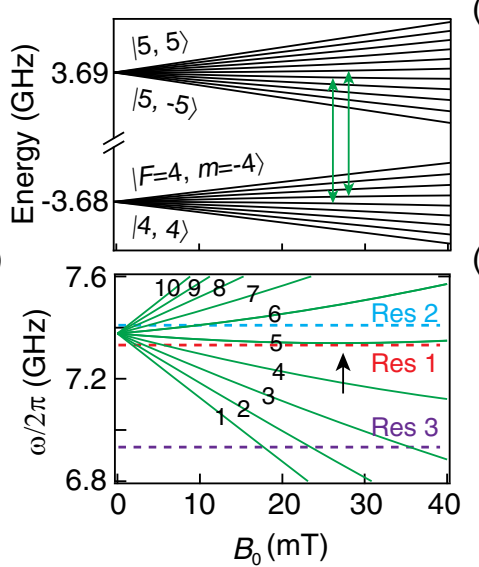

(e)

(f)

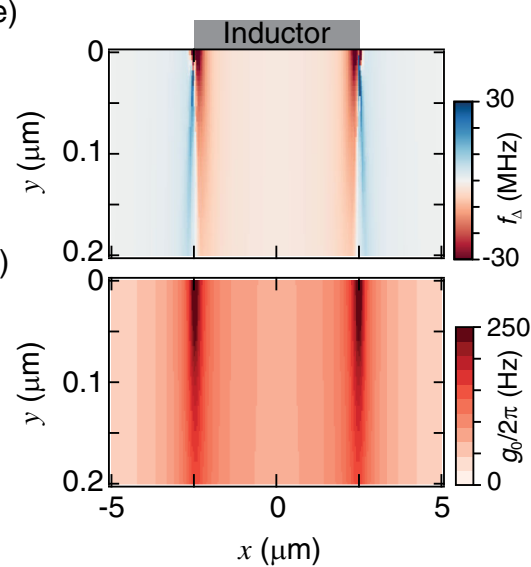

(h)

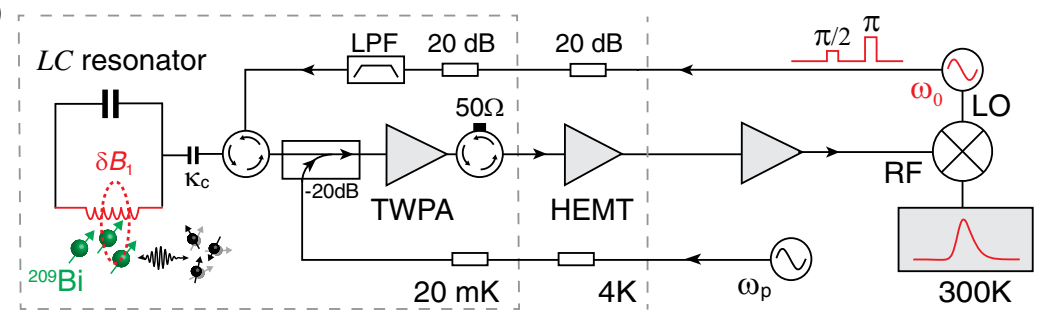

FIG. 1. Bismuth spins in silicon coupled to $L C$ resonators. (a) A schematic of a bismuth atom and associated electron cloud in the silicon lattice. The donor electron stays bound to the $\mathrm{Bi}^{+}$core at low temperatures due to Coulomb potential. (b) Optical picture of one of the $50 \mathrm{~nm}$ thick aluminum $L C$ resonators directly fabricated on the Si substrate ( $x z$ plane and $y=0)$. The inductor is of width $w$ and length $l$, while inter-digitated finger capacitors are $50 \mu \mathrm{m}$ wide and apart. (c) Numerically calculated eigenenergies $|F, m\rangle$ and (d) EPR allowed transition frequencies of Si:Bi at low magnetic fields. Transitions are numbered for referencing. A clock transition near $27 \mathrm{mT}$ is marked by the arrow. (e) Strain induced Larmor frequency shift $f_{\Delta}$ and (f) spin-photon coupling strength $g_{0}$ for the first transition in Res1. (g) The implantation profile $\rho(y)$ of the Bi dopants. (h) The measurement setup at different temperature stages. Input pulses are sent through a series of attenuators and low pass filters (LPF). The reflected output signals are routed via a circulator and amplified with a Josephson traveling wave parametric amplifier (TWPA) before further amplification and demodulation at room temperature. The shallow-implanted Bi spins interact with fluctuating impurity spins on the substrate surface, and are detected via inductive coupling to the resonator.

transition frequencies as a function of $B_{0}$ in the vicinity of the ZFS. Because eight pairs of these transitions are quasidegenerate, only ten curves are resolved. It can be shown that in the low-field limit (except near the clock transition), the transition frequency $\omega(m)$ of $|4, m\rangle \leftrightarrow$ $|5, m+1\rangle$ with $m \in[-4,4]$, as well as $|4, m+1\rangle \leftrightarrow$ $|5, m\rangle$ with $m \in[-5,3]$, is given by

$$
\omega(m) \approx 5 A+\frac{2 m+1}{10} \gamma_{e} B_{0} .
$$

We refer to these ten resonances by the labels $i=$ $1,2, \ldots, 10$ from the lowest to the highest frequency [see Fig. 1(d)]. It is notable that the effective gyromagnetic ratio $\gamma_{\text {eff }} \equiv \mathrm{d} \omega / \mathrm{d} B_{0} \simeq(2 m+1) \gamma_{e} / 10$ strongly depends on $m$ and therefore on $i$. In the following, we exploit the transition-dependent $\gamma_{\text {eff }}$ and hence variable sensitivity to magnetic noise in our analysis of decoherence.

In addition to the obvious sensitivity to magnetic fields via the Zeeman effect, bismuth donor spins are also sensitive to strain (described by the tensor $\varepsilon$ ) and electric field $\mathbf{E}$ via the hyperfine coupling constant $A(\varepsilon, \mathbf{E})$ [22].

Strain modifies the donor wave function and, therefore, also the Fermi-contact hyperfine constant $A$. This effect was quantitatively studied in Ref. [22], where the change $\Delta A(\varepsilon)$ with respect to the unperturbed value $A_{0}$ was found to be

$$
\begin{aligned}
\frac{\Delta A(\varepsilon)}{A_{0}}= & \frac{K}{3}\left(\epsilon_{x x}+\epsilon_{y y}+\epsilon_{z z}\right) \\
& -\frac{L}{2}\left[\left(\epsilon_{x x}-\epsilon_{y y}\right)^{2}+\left(\epsilon_{y y}-\epsilon_{z z}\right)^{2}+\left(\epsilon_{z z}-\epsilon_{x x}\right)^{2}\right],
\end{aligned}
$$

where $\epsilon_{i i}$ are the uniaxial components of the strain tensor, $K=19.1$ is determined by the measurements, and is determined $L=9720$ by the valley repopulation model (VRM) [22]. For strain values less than $10^{-3}$, the hydrostatic component of strain dominates the shift in $\omega(m)$ whose sign, unlike the prediction from the VRM, can be 
both positive and negative. Besides modifying the spin Hamiltonian parameters, strain can also affect the spinlattice relaxation time, as shown recently [17].

The hyperfine coupling constant $A$ is also dependent on an applied electric field $\mathbf{E}$. This Stark effect $\Delta A(\mathbf{E}) / A_{0}=$ $\eta E^{2}$ is quadratic with $\eta=(-0.26 \pm 0.05) \times 10^{-3} \mu \mathrm{m}^{2} / \mathrm{V}^{2}$ for bismuth donors, a value obtained by measurements that is close to the value calculated by a multivalley effective mass theory [30].

Interestingly, because each transition frequency satisfies $\partial \omega / \partial A \simeq 5$ [see Eq. (2)] in the low-field regime, both strain and electric field have a very simple effect on the complex bismuth energy spectrum: They cause a frequency shift $f_{\Delta}(\varepsilon, \mathbf{E})=5[\Delta A(\varepsilon)+\Delta A(\mathbf{E})] / 2 \pi$, which is identical for all bismuth donor transitions.

Another feature of bismuth donor spins relevant to this work is the existence of biasing conditions where $\gamma_{\mathrm{eff}}=0$. Such clock transitions (CT) [10] are the result of the competition between the hyperfine and Zeeman interactions, in a regime where the low-field approximation, Eq. (2), is no longer strictly valid. At a CT, the donor becomes insensitive to magnetic noise to first order, allowing us to probe other sources of noise. In the following, we use the CT that occurs at $27 \mathrm{mT}$ [see Fig. 1(d)] to investigate nonmagnetic sources of decoherence, such as charge noise, which is known to exist in silicon CMOS structures.

\section{MATERIALS AND METHODS}

Our device is fabricated on a silicon (100) chip, having an isotopically purified epitaxial layer of ${ }^{28} \mathrm{Si}(0.05 \%$ of ${ }^{29} \mathrm{Si}$ ), in which bismuth atoms are implanted about $75 \mathrm{~nm}$ below the surface [Fig. 1(e)]. The chip is cleaned in a Piranha solution (3:1 mix of $\mathrm{H}_{2} \mathrm{SO}_{4}$ and $\mathrm{H}_{2} \mathrm{O}_{2}$ at $120^{\circ} \mathrm{C}$ for 15 minutes). Thus, the chip has a native silicon-oxide layer. Finally, three $L C$ superconducting resonators are patterned on top by standard electron-beam lithography, followed by oxygen ashing and evaporation of aluminum. The inductors of the resonators are fabricated along the [011] crystallographic axis ( $z$-frame axis) and have different widths ( $w=5 \mu \mathrm{m}, 2 \mu \mathrm{m}$, and $1 \mu \mathrm{m}$ for Res 1 , Res 2 , and Res3, respectively). The resonance frequencies $\omega_{0}$ are designed to be close to the ZFS for accessing multiple transitions at small magnetic fields and thus preserving low loss rates of the superconducting resonators.

The chip is mounted inside a copper box with a hole and an antenna terminating at a $50 \Omega$ line. The line and the antenna fix the energy decay rates $\kappa_{c}$ of the resonators and allow us to drive and measure the spins in reflection [see Fig. 1(e) and Ref. [23] ]. The copper box is installed inside a homebuilt coil that produces a magnetic field parallel to the inductor. All measurements are performed in a dilution refrigerator at about $15 \mathrm{mK}$ (unless otherwise mentioned), its base temperature. By measuring the reflection coefficient of each resonator with a vector network analyzer, we extract $\kappa_{c}$, the internal loss rate $\kappa_{i}$, and the total loss rate
TABLE I. Resonator parameters.

\begin{tabular}{lccc}
\hline \hline & Res1 & Res2 & Res3 \\
\hline$w(\mu \mathrm{m})$ & 5 & 2 & 1 \\
$l(\mu \mathrm{m})$ & 700 & 450 & 450 \\
$\omega_{0} / 2 \pi(\mathrm{GHz})$ & 7.338 & 7.402 & 6.945 \\
$Z_{0}(\Omega)$ & 40 & 40 & 45 \\
$\kappa_{\mathrm{i}}\left(\mathrm{s}^{-1}\right)$ at $B_{0}=0$ & $4.6 \times 10^{5}$ & $4.6 \times 10^{5}$ & $5.5 \times 10^{5}$ \\
$\kappa_{\mathrm{c}}\left(\mathrm{s}^{-1}\right)$ & $4.6 \times 10^{5}$ & $4.6 \times 10^{5}$ & $1.5 \times 10^{5}$ \\
$\kappa\left(\mathrm{s}^{-1}\right)$ at $B_{0}=0$ & $9.2 \times 10^{5}$ & $9.2 \times 10^{5}$ & $7 \times 10^{5}$ \\
\hline \hline
\end{tabular}

$\kappa=\kappa_{i}+\kappa_{c}$, at an average intracavity photon number $n_{\text {cav }} \sim 1$ (see Table I). All spectroscopy and coherence time measurements are achieved using a two-pulse Hahnecho sequence $\pi / 2-\tau-\pi-\tau-$ echo and computing the echo integral $\chi_{e}$. Control pulses are either square or shaped [31] to exceed the resonator bandwidth limitation. Their absolute amplitude at the level of the resonators is deduced from the Rabi oscillation measurements (Appendix A).

Bismuth spins are inductively coupled to the resonator with a single-spin photon coupling strength, $g_{0}=$ $\gamma_{e}\left\langle 4, m\left|S_{x}\right| 5, m \pm 1\right\rangle \cdot \delta B_{1}$, where $\delta B_{1}$ is the rms vacuum fluctuation of the resonator magnetic field [23]. The corresponding Rabi frequency is given by $\Omega_{\mathrm{R}}=2 g_{0} \sqrt{n_{\text {cav }}}$. We calculate $\delta B_{1}$ for different resonators using finite element simulations by solving for a current $\delta i=\omega_{0} \sqrt{\hbar / 2 Z_{0}}$ flowing through the inductor with an appropriate spatial density in the superconducting film [32]. Here, $Z_{0}$ is the impedance of the resonator estimated independently from electromagnetic simulations.

One specificity of our setup is the small mode volume of the superconducting resonators, enabling $g_{0} / 2 \pi$ to reach large values $10^{2}-10^{3} \mathrm{~Hz}$. This enhances the spin-radiativeenergy relaxation rate via the Purcell effect [33-35], at a rate $\Gamma_{\mathrm{P}}=4 g_{0}^{2} / \kappa$ for resonant spins. Because of the Purcell effect, spins have $T_{1}$ times in the order of a second or lower (see below) instead of hours [9,34], which makes signal averaging possible. Another specificity is the use of a superconducting parametric amplifier [36] to amplify the echo signal, which results in detection sensitivities in the $10-10^{3}$ spins $/ \sqrt{\mathrm{Hz}}[23-25]$ range. On the other hand, the use of planar microresonators leads to large spatial inhomogeneity of the $B_{1}$ field and thus also of $g_{0}$, as shown in Fig. 1(f). The resulting spatially inhomogeneous Rabi frequency and spin relaxation times lead to a complex echo response when subject to varying pulse amplitudes and repetition rates of the measurement [37]. Some of these complex responses are described further below.

\section{SPECTROSCOPY}

The spectrum of bismuth donors in silicon coupled to an aluminum superconducting microresonator patterned on top was measured recently [26] in a geometry very similar 
(a)

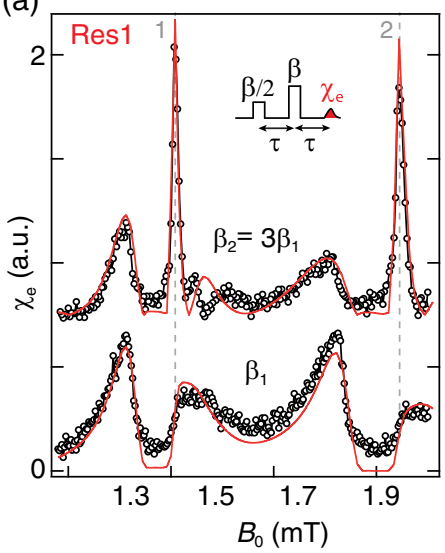

(b)

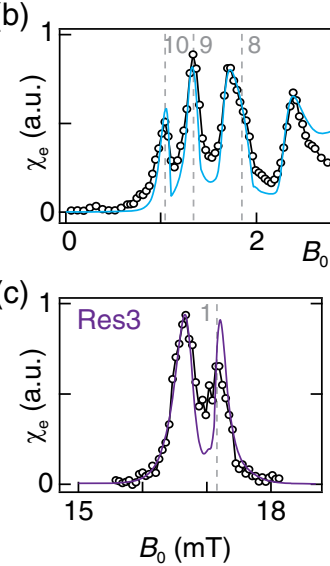

(e)

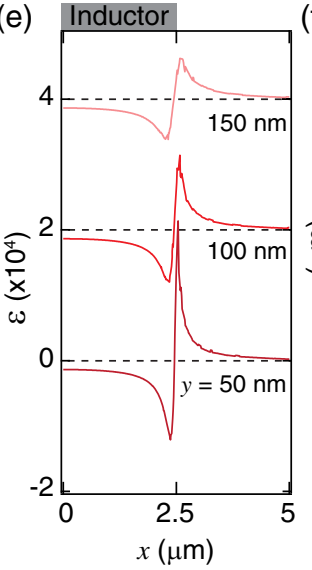

(f)

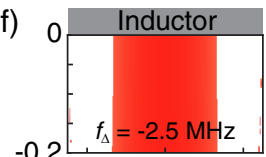

$-0.2$
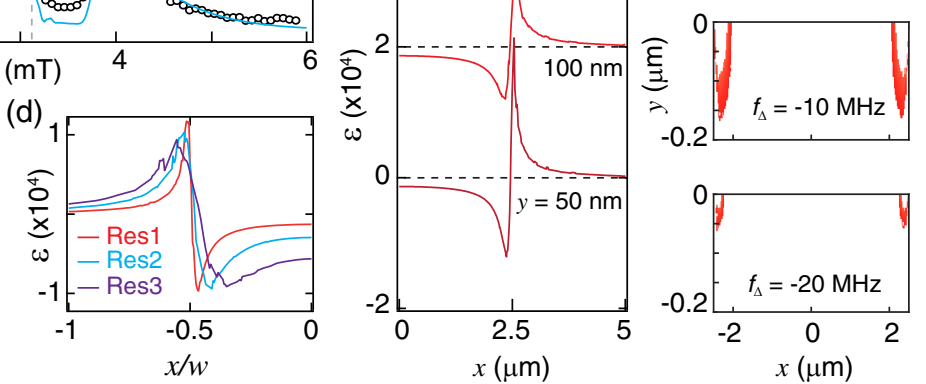

FIG. 2. Strain spectroscopy. (a)-(c) Measured (symbols) and numerically modeled (solid lines) echo-detected spin spectroscopy for three resonators. The Hahn-echo sequence consists of square pulses of amplitudes $\beta / 2, \beta$, which are each $2 \mu$ s long and averaged at a repetition rate of $10 \mathrm{~s}(\operatorname{Res} 1), 2 \mathrm{~s}(\operatorname{Res} 2)$, and $0.5 \mathrm{~s}(\operatorname{Res} 3)$. The area under the echo is labeled as $\chi_{e}$. Vertical dashed lines mark the position of spin transitions in the absence of any strain. The transition numbers $(i=1,2, \ldots, 10)$ are marked as described in Fig. $1(\mathrm{~d})$. In panel (a), the top (bottom) measurement is done at a pulse amplitude $\beta_{2}=15 \times 10^{6} \mathrm{~s}^{-1 / 2}\left(\beta_{1}=5 \times 10^{6} \mathrm{~s}^{-1 / 2}\right)$. (d) Comparison of the hydrostatic component of strain $\varepsilon=\left(\epsilon_{x x}+\epsilon_{y y}+\epsilon_{z z}\right) / 3$ at a fixed depth $y=75 \mathrm{~nm}$ across resonators and (e) at different depths in Res 1 . Curves have been offset by $2 \times 10^{-4}$ for clarity. (f) Inferred location of spins (from COMSOL simulation) for different $f_{\Delta}$ within a square bandwidth of $\pm 0.2 f_{\Delta}$ in Res1.

to the one used in our device. The spectrum was shown to be strongly distorted by strain in the substrate resulting from differential thermal contractions between the resonator and the silicon. The aim of this section is to support these earlier findings with complementary measurements performed on resonators having a different geometry.

Strain arises during cooldown from room temperature, at which the device is fabricated, to millikelvin temperatures, at which it is measured. The strain tensor $\varepsilon$ can be calculated using finite element simulations from COMSOL [26,38], with the known parameters for the differential thermal contraction of silicon relative to aluminum (see Appendix B). From the strain tensor, the frequency shift $f_{\Delta}(\varepsilon)$ is computed using Eq. (3). An essential aspect of the present measurements is that, because of the strain gradient, $f_{\Delta}(x, y)$ depends on the location $(x, y)$ of the donor. It also strongly depends on the width of the inductive wire, as can be seen in Fig. 2(d).

The computed strain-induced frequency shift $f_{\Delta}(x, y)$ is shown in Fig. 1(e) for the geometry of Res1. The values are in the $1-10-\mathrm{MHz}$ range, much larger than both the resonator linewidth $(\kappa / 2 \pi \sim 150 \mathrm{kHz})$ and the linewidth of about $100 \mathrm{kHz}$ [39] due to the dipolar interaction with the bath of residual ${ }^{29} \mathrm{Si}$ nuclear spins. Therefore, the bismuth donor spin spectrum is expected to be entirely dominated by strain shifts, as confirmed experimentally in the following. We also note that the strain, and thus $f_{\Delta}(x, y)$, only weakly depends on $y$ unless $x$ is close to $\pm w / 2$ [Figs. 2(e) and 2(f)].

Echo-detected spectra are measured with the sequence shown in the inset of Fig. 2(a), consisting of two square pulses of amplitude $\beta / 2$ and $\beta$ and duration $t_{p}$, separated by a delay $\tau$, giving rise to an echo after a further delay $\tau$. The sequence is performed with a repetition time greater than or equal to $3 T_{1}$. The integral $\chi_{e}$ of the resulting echo is plotted as a function of $B_{0}$ in Fig. 2 for the three resonator geometries. Moreover, for Res1, spectra are acquired with two different pulse amplitudes $\beta$.

We first notice that each spectral feature in Fig. 2(a) is composed of two split peaks. Moreover, the right peak amplitude strongly depends on $\beta$, contrary to the left peak whose amplitude is barely changed. These observations can be qualitatively understood as follows. Spins located below the inductor have negatively shifted $f_{\Delta}$ and form the lowfield peak. These have larger $g_{0}$ than those far away from the wire, and they undergo Rabi angles close to $3 \pi$ and $\pi$ for the two chosen $\beta$ values, leading to similar peak amplitudes. On the other hand, the high-field peaks are formed by weakly strained spins $\left(f_{\Delta} \sim 0\right)$ located far from the wire; thus, they appear near the frequency of the unperturbed donor spectrum. Because of the weaker $g_{0}$, such distant spins undergo smaller Rabi angles than in the left peak, closer to $\pi$ and $\pi / 3$, respectively, leading to very different echo amplitudes. The low-field (high-field) tails are formed by spins near the inner (outer) edges of the inductor that are also the most strained ones and closer to the sample surface.

Spectra acquired with Res 2 and Res 3 are presented in Figs. 2(b) and 2(c). We again observe a split-peak behavior, though there are significant overlaps in the case of Res2 due to the close spacing of the peaks. Another observation is that the peak splitting progressively increases from Res 1 to Res3. For example, in Res3, the splitting is about $0.5 \mathrm{mT}$ compared to about $0.1 \mathrm{mT}$ in Res 1 . This case is readily explained by the value of the hydrostatic strain at $x=0$, 
which is indeed about 5 times larger in Res 3 than in Res1 [Fig. 2(d)]. Another visible feature in the measurements is the dependence of the linewidth on the transition. This feature is also consistent with strain broadening, which causes a frequency broadening $\Delta \omega=(\partial \omega / \partial A) \Delta A$, uniform across all transitions [see Eq. (2)], and therefore a transition-dependent peak width of $\Delta \omega / \gamma_{\text {eff }}(i)$ upon a magnetic-field sweep. Note that Res2 frequency is larger than the ZFS, so the split peaks are inverted compared to Res 1 and Res3; i.e., spins below the wire now give rise to the high-field peaks, and spins away from the wire give rise to the low-field peaks.

To go beyond these qualitative arguments, a simple model is developed. It incorporates two physical effects: the spatial inhomogeneity of the strain shift $f_{\Delta}(x, y)$ and of the coupling constant $g_{0}(x, y)$, which leads to a spatially inhomogeneous Rabi angle $\theta(x, y)=4 g_{0} \beta / \sqrt{\kappa} t_{p}$ and Purcell relaxation rate $\Gamma_{P}(x, y)$. We compute the echo amplitude at a certain field $B_{0}$ due to transition $i$ as follows:

(i) Compute the corresponding shift $f_{\Delta}\left(i, B_{0}\right)=$ $\left[\omega_{0}-\omega_{i}\left(B_{0}\right)\right] / 2 \pi$.

(ii) Determine the total areas $\mathcal{A}\left(i, B_{0}\right)$ of the regions in the $x y$ plane, where the strain-induced shift is comprised between $f_{\Delta}\left(i, B_{0}\right)-\kappa /(2 \pi)$ and $f_{\Delta}\left(i, B_{0}\right)+$ $\kappa /(2 \pi)$.

Examples in Fig. 2(f) show that for larger values of $f_{\Delta}\left(i, B_{0}\right)$, the areas $\mathcal{A}\left(i, B_{0}\right)$ are closer to the edges of the wire and also to the sample surface because strain increases close to the wire edges and substrate surface, as is apparent in Figs. 2(e) and 2(f). Therefore, donors that display a large strain shift are only found in the area close to the wire edge and sample surface.

(iv) Compute the echo amplitude due to transition $i$ as

$$
\begin{aligned}
\chi_{e}\left(i, B_{0}\right)= & 2 \frac{\sqrt{\kappa_{\mathrm{c}}}}{\kappa} \iint_{\mathcal{A}\left(i, B_{0}\right)} d x d y l \rho(y) g_{0}(x, y) \\
& \times\left[1-\exp \left(-\Gamma_{\mathrm{P}}(x, y) t_{\mathrm{rep}}\right)\right] \sin ^{3}[\theta(x, y)],
\end{aligned}
$$

where $\rho(y)$ is the implanted bismuth donor density and $l$ the wire length [37].

(v) We account for a possible inhomogeneity of the strain field by convolving the result with a Gaussian $\mathcal{C}(x)=\left(1 / \sigma_{B} \sqrt{2 \pi}\right) \exp \left(-x^{2} / 2 \sigma_{B}^{2}\right)$ of standard deviation $\sigma_{B}$ :

$$
\tilde{\chi}_{e}\left(i, B_{0}\right)=\int \chi_{e}(i, B) \mathcal{C}\left(B_{0}-B\right) d B
$$

(vi) The resulting echo amplitudes are finally summed over all transitions, with individual weights $p_{i}$ :

$$
\chi_{e}\left(B_{0}\right)=\sum_{i} p_{i} \tilde{\chi}_{e}\left(i, B_{0}\right) .
$$

This model is slightly more complete than the one used in Ref. [26], as it takes into account the spectrum distortions due to the finite repetition time and to the spatial distribution of the Purcell relaxation rate [37]. The adjustable parameters are the local strain inhomogeneity $\sigma_{B}$ and the relative weights $p_{i}$ of the various transitions. Moreover, we allow ourselves to slightly adjust the $\mathrm{Al}$ deposition temperature in the strain calculation for each resonator geometry to match the measurements best. In Ref. [26], the implanted donor density $\rho(y)$ was, moreover, multiplied by the probability that a donor is ionized because of the Schottky barrier formed between the aluminum and the silicon, which leads to the formation of a depletion area below the wire. Here, we find a better agreement if we consider that the depletion area is of negligible extent, as shown in Appendix C. This result could be due to the existence of positive charges in the native silicon-oxide layer or to the exceedingly long time it takes to reach charge equilibrium. Based on this observation, we entirely neglect the possible existence of a Schottky barrier in all the calculations described below. More work would be needed to determine the characteristics of the contact interface between aluminum and silicon in our device.

The result of the spectrum calculations is shown in Fig. 2 for all resonator geometries, after optimization of the adjustable parameters. We find that a good agreement is obtained for all resonator geometries by taking $\sigma_{B}=$ $0.2 f_{\Delta}\left(i, B_{0}\right) / \gamma_{\mathrm{eff}}$, suggesting that the strain tensor displays some amount of fluctuations around the value obtained by modeling the thin film and substrate with a continuous medium theory. The next section will prove that this inhomogeneity of about $20 \%$ of the strain field is actually local and happens on a scale of about $50 \mathrm{~nm}$, which may be linked to the polycrystalline nature of the aluminum thin film.

The model semiquantitatively reproduces the two spectra measured on Res1 for different pulse amplitudes, for an initial temperature of $300 \mathrm{~K}$. It also reproduces most of the spectral features measured for Res 2 and Res3, for a deposition temperature of, respectively, $270 \mathrm{~K}$ and $250 \mathrm{~K}$, reasonably close to room temperature. The difference in deposition temperature may be due to the fact that the native silicon oxide is not included in the strain calculation. Interestingly, we need to assume different relative individual weights $p_{i}$ to reproduce the spectra: $p_{1}=1, p_{2}=1.2$ for Res 1 , and $p_{10}=1, p_{9}=1.2, p_{8}=1.5, p_{7}=1.8$ for Res2, whereas all $p_{i}$ should be approximately equal at thermal equilibrium at $20 \mathrm{mK}$. An out-of-equilibrium distribution of hyperfine states is not surprising, considering that the phonon relaxation rates in between these levels become exceedingly long at low temperatures (likely on the order of days). First, we note that, because the peaks are not distorted but simply multiplied by a weighting factor, the 
observed hyperfine population imbalance cannot be caused by or even correlated to the strain profile in our sample. On the other hand, above-gap infrared radiation has been shown to efficiently induce hyperpolarization among the hyperfine sublevels of donors in silicon [40]; therefore, stray infrared photons reaching our sample and originating from higher temperature stages in the cryostat may be the cause of the observed hyperfine imbalance, similar to recent observations of ultraslow and out-of-equilibrium dynamics between hyperfine sublevels of bismuth donors in silicon [41].

The quantitative understanding of the spectra achieved for various resonator geometry and pulse amplitudes confirms the results presented in Ref. [26], stating that strain shifts are the dominant broadening mechanism in this kind of device. The refined analysis enables us to find two new features: (1) The strain tensor appears to fluctuate by about $20 \%$ around the value determined by finiteelement modeling with a continuous-medium approach; it is tempting to link this inhomogeneity with the polycrystalline nature of the aluminum film, which is not taken into account in the modeling. (2) The population distribution in the hyperfine manifold appears to be out of equilibrium. In the rest of this work, we leverage the correlation between position in the spectrum [corresponding to a given strain shift $f_{\Delta}\left(B_{0}\right)$ ] and spatial location [the area $\left.\mathcal{A}\left(i, B_{0}\right)\right]$ to obtain spatially resolved information on the donor coherence properties and therefore on the noise they experience. However, we note that the spatial resolution is mainly in the lateral dimension $x$ since strain is only weakly dependent on $y$, except close to the wire edges.

\section{INSTANTANEOUS DIFFUSION}

In this section, we use the phenomenon of instantaneous diffusion (ID) to demonstrate that the $20 \%$ strain inhomogeneity evidenced by the spectral measurements described in the previous section is local.

Various decoherence mechanisms for dilute paramagnetic impurities arise through dipolar interactions with other spins. Dipolar interactions between some "central spins" and its neighbors can be refocused using a Hahn-echo sequence $\pi / 2-\tau-\pi-\tau-$ echo; however, if the neighboring spins also fall within the bandwidth of the $\pi$ pulse, they are also flipped and the sign of the dipolar interaction term is not reversed. The effect of such dipolar interactions between similar spins can be probed by repeating the Hahn-echo sequence using a smaller refocusing angle $\theta$, which can be interpreted as flipping only a fraction of spins. At the cost of a reduced echo intensity [due to the reduced probability $\sin ^{2}(\theta / 2)$ of flipping the central spin], the dipolar interaction with its neighbors is correspondingly refocused. The resulting coherence decay time constant $T_{2}$ thus follows [42]:

$$
1 / T_{2}=\Gamma_{\text {res }}+\rho \gamma_{\text {eff }}^{2} \frac{\pi \hbar \mu_{0}}{9 \sqrt{3}} \sin ^{2}\left(\frac{\theta}{2}\right)
$$

where $\rho$ is the number of spins per unit volume and $\Gamma_{\text {res }}$ the residual decoherence rate due to other spectral diffusion or decoherence mechanisms. Measuring the dependence of $T_{2}$ on $\theta$ thus provides a convenient method to determine the sample concentration $\rho$. Note that local inhomogeneous broadening tends to suppress ID, if this broadening is comparable to or larger than the pulse excitation bandwidth. Here, we measure ID for various pulse excitation bandwidths and extract some information about local inhomogeneous broadening.

For this purpose, we select spins located in the middle of the wire of Res1 [left peaks in Fig. 2(a), corresponding to $f_{\Delta}=-2.5 \mathrm{MHz}$. Well-defined Rabi oscillations are obtained, enabling quantitative knowledge of $\theta$ (see Appendix A). The coherence time $T_{2}$ is measured by varying the delay $\tau$ in a Hahn-echo sequence and fitting an exponential $\exp \left(-2 \tau / T_{2}\right)$ to the measured decay of the echo area $\chi_{e}$. The decoherence rate $1 / T_{2}$ is shown in Fig. 3(a) as a function of $\sin ^{2}(\theta / 2)$, for different transitions and pulse excitation bandwidth $\Omega$. A linear dependence is found in all cases, indicative of ID. For a given bandwidth $\Omega /(2 \pi)=1 \mathrm{MHz}$, the ratio of slopes between the two transitions matches the expected $\gamma_{\text {eff }}^{2}$ dependence characteristic of ID [Eq. (7)]. The slope is seen to strongly depend on $\Omega$ [Fig. 3(a)], which we interpret as evidence for inhomogeneous broadening on a length scale corresponding to the distance between neighboring donors in the same level (thus, susceptible to be flipped by the same control pulse), which, in our case, is about $50 \mathrm{~nm}$. Note that because of the limited microwave power, it i impossible to increase $\Omega / 2 \pi$ (a)

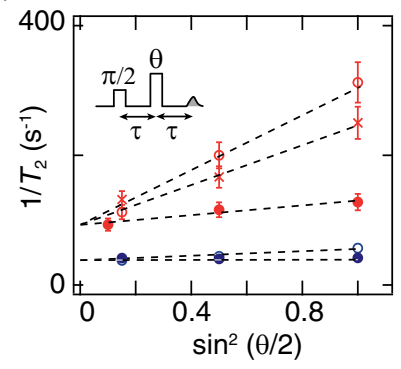

(b)

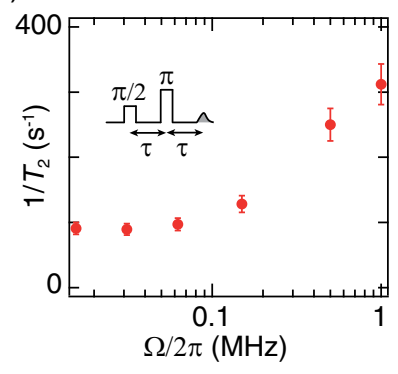

FIG. 3. Instantaneous diffusion in Res1. (a) Measured echo amplitude decay rate $T_{2}^{-1}$ as a function of $\sin ^{2}(\theta / 2)$, with $\theta$ being the Rabi angle achieved during the refocusing pulse, for the first transition (red symbols) and the fourth transition (blue symbols). Solid circles, crosses, and open circles are data for refocusing pulse bandwidths of $150 \mathrm{kHz}(\kappa / 2 \pi), 500 \mathrm{kHz}$, and $1 \mathrm{MHz}$, respectively. Dashed lines are linear fits to the data. (b) Decay rate $T_{2}^{-1}$ of the echo amplitude as a function of the pulse bandwidth $\Omega$, for transition $i=1$, and at a refocusing angle of $\pi$. Measurements are done with square pulses of duration $64 \mu \mathrm{s}, 32 \mu \mathrm{s}, 16 \mu \mathrm{s}$, and $2 \mu \mathrm{s}$, and shaped pulses of duration $0.5 \mu \mathrm{s}$ and $1 \mu \mathrm{s}$. 
above $1 \mathrm{MHz}$, but ID keeps getting stronger up to that value, indicating that the broadening can be greater than or equal to $1 \mathrm{MHz}$.

Sources of local broadening include the Overhauser field due to the residual ${ }^{29} \mathrm{Si}$ nuclear spins, which should be of order $100 \mathrm{kHz}$ given the $5 \times 10^{-4}$ relative ${ }^{29} \mathrm{Si}$ concentration, significantly lower than what is needed to explain the data in Fig. 3(b). Local electric-field variations would need to be excessively large to explain our data and are thus unlikely. We believe that local variations of the strain tensor are the most likely explanation for our data, especially since strain inhomogeneity is also required to quantitatively fit the spectra, as explained in the previous section. The polycrystalline nature of the aluminum film (with grain size on the order of tens of nanometers) may be the cause of the strain tensor variations. If this is indeed the case, such local variations should be a general phenomenon occurring below all superconducting devices since they are, in general, patterned out of polycrystalline films (aluminium, niobium, niobium nitride, etc.), with the magnitude of strain inhomogeneity depending on the film material and thickness. Studying instantaneous diffusion of paramagnetic impurities - and, in particular, of bismuth donors in silicon because of their exceptionally strong sensitivity to strain-is evidently a powerful technique for characterizing strain variations on a local scale.

Using Eq. (7), we extract from the data of Fig. 3(a) (at $\Omega / 2 \pi=1 \mathrm{MHz}$ ) a concentration $\rho=5 \times 10^{14} \mathrm{~cm}^{-3}$ for bismuth donor spins in level $|4,-4\rangle$. This value is still an order of magnitude lower than the one expected from the nominal average bismuth concentration assuming a thermal distribution within the nine hyperfine levels of the groundstate manifold. We attribute this difference to a combination of finite donor activation yield [39], insufficiently large $\Omega$ to fully compensate the strain inhomogeneity, and out-ofequilibrium distribution of the donors within the hyperfine manifold (as seen in Sec. IV).

\section{DECOHERENCE DUE TO MAGNETIC NOISE}

We now leverage the transition- and magnetic-fielddependent $\gamma_{\text {eff }}(i)$ [see Fig. 1(d) and Eq. (2)], as well as the correlation between spatial position and strain-induced frequency shift, to quantify the magnetic noise seen by bismuth donor spins, and we obtain information about its spatial origin. In the following, all measurements are done with pulses limited by the resonator bandwidth $(\Omega / 2 \pi \sim 0.15 \mathrm{MHz})$, such that the effect of ID is less than 20\% (see Fig. 3). Moreover, we adjust the repetition time to always be longer than $3 T_{1}$ (see Appendix A) so that the spins are fully polarized for each measurement discussed below.

Using Res1, we first compare the coherence time $T_{2}$ obtained across different transitions, from donor spins located in a region characterized by a given strain shift $f_{\Delta}$. Figure 4(a) shows the Hahn-echo integrals $\chi_{e}(2 \tau)$ on transitions $i=1$ to 4 , at $f_{\Delta}=-2.5 \mathrm{MHz}(\langle x\rangle \sim 0.5 \mu \mathrm{m})$. Exponential decays are systematically observed. The extracted exponential decay constant $T_{2}$ shows an increase from $7.5 \mathrm{~ms}\left(i=1, \gamma_{\mathrm{eff}} / \gamma_{e}=0.9\right)$ to $24 \mathrm{~ms}(i=4$, $\gamma_{\text {eff }} / \gamma_{e}=0.3$ ) [see Fig. 4(b)]. The same comparison is performed for two other values of $f_{\Delta}$, corresponding to spins below the wire but not in the middle $\left(f_{\Delta}=-4 \mathrm{MHz}\right.$, $\langle x\rangle \sim 1.5 \mu \mathrm{m}$ ), and as far from the wire as possible $\left(f_{\Delta} \simeq 0,\langle x\rangle \rightarrow \infty\right)$. As seen in Fig. 4(b), we find that $T_{2}^{-1}$ linearly depends on $\gamma_{\text {eff }}$ for each of the three locations, (a)

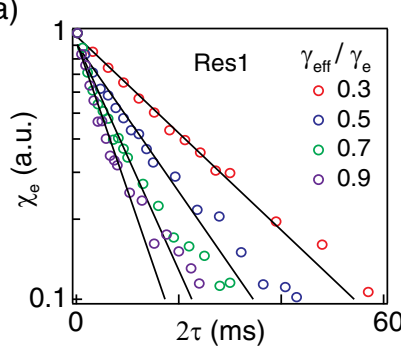

(c)
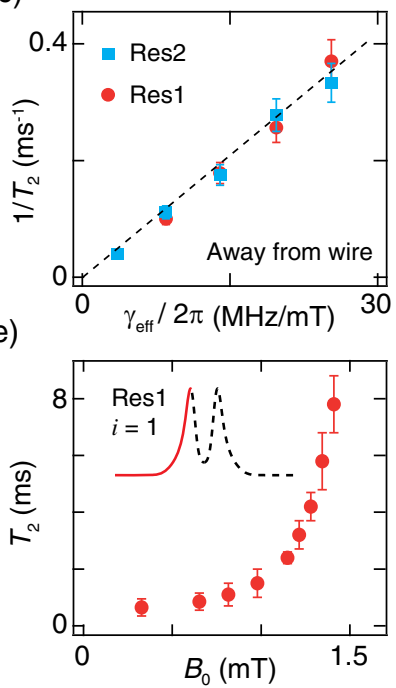

(b)

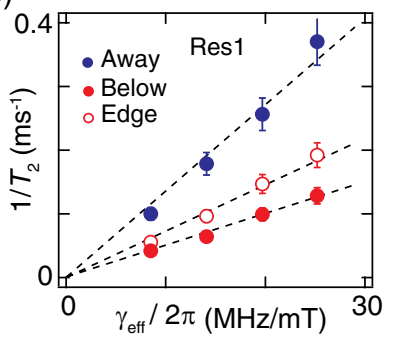

(d)

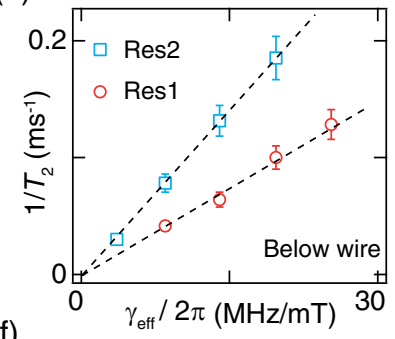

(f)

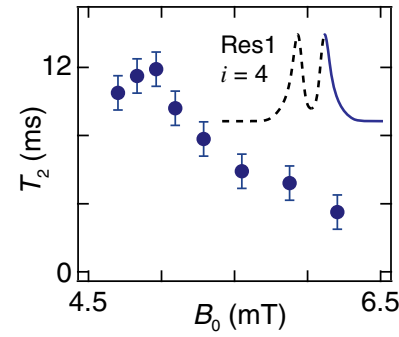

FIG. 4. Spatial magnetic noise. (a) Echo integral $\chi_{e}$ as a function of the delay $2 \tau$ between the $\pi / 2$ pulse and the echo, measured with Res1 for transitions $i=1-4$, at fields $B_{0}$ corresponding to the same $f_{\Delta}=-2.5 \mathrm{MHz}$ (i.e., $\langle x\rangle \sim 0.5 \mu \mathrm{m}$ ). Open circles are the data, and solid lines are exponential fits yielding the decay rate $T_{2}^{-1}$. (b) Extracted echo amplitude decay rate $T_{2}^{-1}$ with Res 1 as a function of $\gamma_{\text {eff }}$ for transitions $i=1-4$ and $f_{\Delta}=$ $-2.5 \mathrm{MHz}$ (spins below the wire, red dots), $f_{\Delta}=-4 \mathrm{MHz}$ (spins closer to the wire edge, red circles), and $f_{\Delta}=0 \mathrm{MHz}$ (spins far away from the wire, blue dots). The dashed lines are linear fits. (c, d) Comparison between resonators. Echo amplitude decay rate $T_{2}^{-1}$ as a function of $\gamma_{\text {eff }}$ measured with Res1 (transitions $i=1-4$, red dots) and with Res2 (transitions $i=6-10$, blue squares). Panel (c) shows the data for $f_{\Delta}=0$ (spins far away from the wire) and panel (d) for spins below the wire. (e) Coherence time $T_{2}$ measured with Res1 as a function of $B_{0}$ on the low-field peak of the $i=1$ transition. (f) Coherence time $T_{2}$ measured with Res 1 as a function of $B_{0}$ on the high-field peak of the $i=4$ transition. 
with linear extrapolation at $\gamma_{\text {eff }}=0$ very close to 0 . This finding establishes that the dominant source of decoherence on the transitions considered here is of magnetic origin, for all three spin locations.

Without having to assume any decoherence model, we can reexpress the measured coherence times $T_{2}$ into an effective magnetic noise by computing $\delta B=2 \pi /\left(\gamma_{\text {eff }} T_{2}\right)$. As seen from the line slopes in Fig. 4(b), we observe a marked dependence of $\delta B$ on the location of the probed donor spins relative to the wire and substrate surface. Complementary measurements are provided by comparing $\delta B$ in Res1 and Res2. As seen in Fig. 4(c), $\delta B$ is found to be the same in Res1 and Res2 for spins away from the wire $\left(x \rightarrow \infty, f_{\Delta} \simeq 0\right)$, whereas $\delta B$ is twice as large in Res 2 as in Res1 for spins at $\langle x\rangle \sim 0.5 \mu \mathrm{m}$, right below the wire. To obtain a comprehensive measurement of $\delta B$, we measure $T_{2}$ as a function of $B_{0}$ on the edge of the low-field peak on transition $i=1$ [Fig. 4(e)] and on the edge of the highfield peak of transition $i=4$ [Fig. 4(f)], as the latter has negligible overlap with other transitions; $T_{2}$ is seen to vary by as much as 1 order of magnitude. Similar results are obtained with Res 2 and Res3. Using our knowledge of $\mathcal{A}\left(i, B_{0}\right)$, we obtain a spatial mapping of $T_{2}$ (or, equivalently, of the noise $\delta B$ ). Figure 5(a) shows the 2D map of $T_{2}$ for Res1 on the transition $i=1$; Fig. 5(c) [respectively, 5(d)] shows the magnetic noise $\delta B$ as a function of the average lateral position $\langle x\rangle$ [respectively, average depth $\langle y\rangle]$. A clear trend emerging from Figs. 5(c) and 5(d) is that the magnetic noise $\delta B$ increases for spins closer to the wire edge, and to the sample surface.

The observation that the magnetic noise $\delta B$ causing bismuth donor spin decoherence is spatially dependent is one of the main results of our work, as it has important implications on the possible physical origin of this noise. Magnet noise, which is a common decoherence mechanism [5], is incompatible with our measurements because it would act uniformly on all donor spins. Another common mechanism is spectral diffusion caused by a spin bath present in the bulk of the sample. The ${ }^{29} \mathrm{Si}$ nuclear spin bath is uniformly distributed in the bulk, and its properties do not depend on strain or position in the sample; it is thus also incompatible with our data.

Spectral diffusion can also be caused by a bath of unpolarized paramagnetic impurities, which induce magnetic noise at the donor spin location because of spin flips due to either spin-lattice relaxation or flip-flops between spin pairs. Our observations shed light on the properties of this bath and help identify its nature. From the data in Fig. 5, we see that the noise is maximum in areas where the strain is maximum, which happen to be close to the wire edge and to the substrate surface. These observations are difficult to explain, considering a paramagnetic impurity bath uniformly distributed in the bulk. Indeed, low levels of strain as encountered here (in the $\sim 10^{-4}$ range) may cause small shifts of the paramagnetic impurity resonance (a)

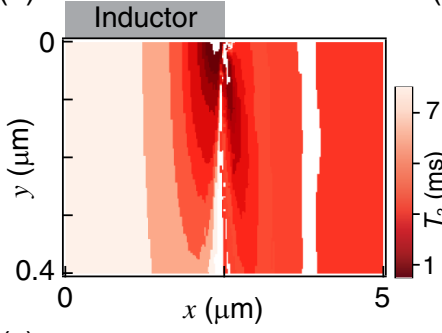

(b)

(c)

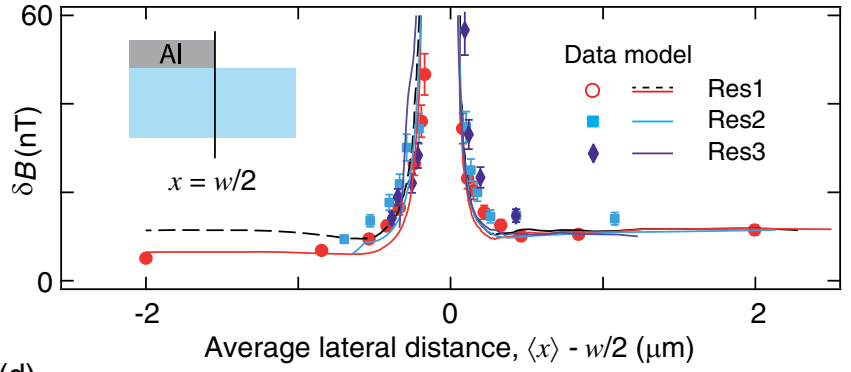

(d)

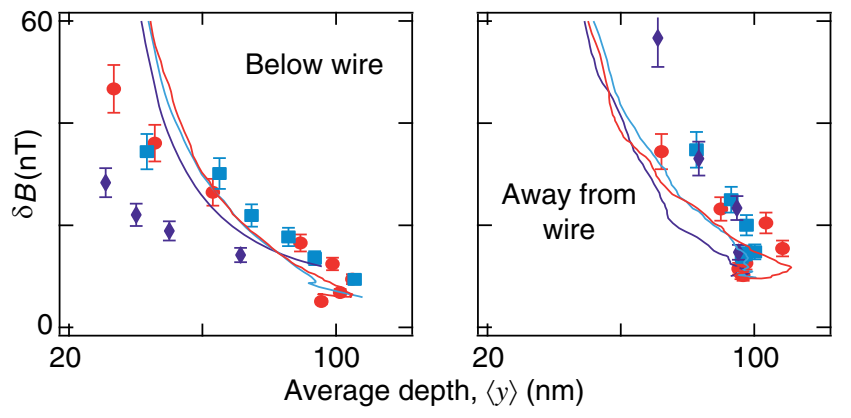

FIG. 5. Spatially dependent magnetic noise. (a) Measured coherence time $T_{2}$ of the first transition $(i=1)$ versus the inferred spin locations from $\mathcal{A}\left(B_{0}\right)$ in Res1. (b) Reconstructed surface magnetic impurity distribution $\sigma$ as a function of the position $x$ with respect to the wire center for the three resonators. $(\mathrm{c}, \mathrm{d})$ Magnetic noise magnitude for the three different resonators as a function of the average spin position inferred from $\mathcal{A}\left(B_{0}\right)$ and the implantation profile. Measurements are colored symbols, and the model described in the text is shown by solid lines. The dashed black curve in panel (c) is computed for Res1 assuming a uniform density $\sigma=4 \times 10^{12} \mathrm{~cm}^{-2}$.

frequency (e.g., in the MHz range for Bi:Si donors), which would then detune paramagnetic impurity pairs and reduce spectral diffusion; thus, one would find the longest donor coherence times in the most highly strained regions, contrary to our observations. Moreover, EPR spectra acquired on samples identical to the ones measured here [39] only detect bismuth donors and dangling bonds and no extra bulk paramagnetic impurity.

On the other hand, the measurements of Figs. 5(c) and 5(d) are compatible with a paramagnetic impurity bath residing at the sample surface, which would naturally explain the stronger noise $\delta B$ for lower average depth $\langle y\rangle$. Such a spin bath likely consists of dangling bonds at the interface between silicon and silicon oxide (the $P_{\mathrm{b}}$ defects), which 
are indeed detected in the EPR spectra [39]. Donor spin decoherence by dangling bonds is an established phenomenon, which was discussed in Ref. [15] and reported in Refs. $[14,16]$.

Decoherence of a spin located at a depth $d$ below a noisy surface containing a surface density $\sigma$ of fluctuating spins was considered in several contexts. The model of de Sousa [15] considers the decoherence of donors in silicon caused by spin-lattice relaxation of paramagnetic $P_{\mathrm{b}}$ defects at the silicon/silicon-oxide interface. It predicts that the echo of an isolated donor with gyromagnetic ratio $\gamma$ should have a Gaussian decay $\chi_{e}(2 \tau) / \chi_{e}(0)=\exp \left[-\left(2 \tau / T_{2}\right)^{2}\right]$, with $T_{2}=K /[\gamma \delta b(d)], \delta b(d)$ being the standard deviation of the magnetic field generated by a distribution of random surface spins at the depth $d$, and $K \sim 10$ a dimensionless parameter linked to the specific modeling of the paramagnetic defect distribution. It can be shown that $\delta b(d)$ scales like $\sqrt{\sigma} / d^{2}$. The less-specific model of Yoneda et al. [43] assumes that the surface spins cause a magnetic noise with power spectrum $S(\omega)=S_{0}(d) / \omega^{\alpha}$. In our case, we have evidence that $\alpha \simeq 1$ (see Sec. VIII), which should also lead to a Gaussian Hahn-echo decay with $T_{2}=$ $2 \pi /\left[\gamma \sqrt{S_{0}(d)}\right][43,44]$. Because of the $1 / f$ divergence at low frequencies, in this model, it is not, strictly speaking, possible to define a magnetic-field-noise standard deviation; nevertheless, after introducing proper infrared cutoff frequencies for the noise, one finds that, within a factor of order unity, $S_{0}(d) \sim \delta b^{2}(d)$. In summary, both models predict a Gaussian-shaped decay, with $T_{2} \sim 2 \pi /[\gamma \delta b(d)]$ within a factor of order unity, which we will disregard in the following.

In our device, the interface cannot be considered homogeneous because of the metallic wire, and two areas can be distinguished. Away from the wire, the interface is between the silicon substrate and the native silicon oxide, whereas below the wire, the interface is more complex, with the 50 -nm-thick aluminum wire on top of the $\mathrm{Si} / \mathrm{SiO} 2$ interface. This case may lead to differences in surface spin density, which we take into account in a minimal model where the surface spin density evolves from $\sigma_{1}$ away from the wire to $\sigma_{2}$ below the wire, with a smooth interpolation of characteristic width $500 \mathrm{~nm}$. We numerically estimate the resulting magnetic noise by discretizing the surface spins into point dipoles of magnitude Bohr magneton $\left(\mu_{\mathrm{B}}\right)$ with random orientation and summing the squares of the magnetic-field component along the applied-field $z$ direction, thus obtaining the rms magnetic noise $\delta b(x, y)$. The echo amplitudes for a given $f_{\Delta}$ and delay $2 \tau$ are then numerically calculated using

$$
\begin{aligned}
\chi_{e}\left(f_{\Delta}, \tau\right)= & 2 \frac{\sqrt{\kappa_{\mathrm{c}}}}{\kappa} \iint_{\mathcal{A}\left(i, B_{0}\right)} d x d y l \rho(y) g_{0}(x, y) \\
& \times\left\{1-\exp \left[-\Gamma_{\mathrm{P}}(x, y) t_{\mathrm{rep}}\right]\right\} \\
& \times \exp \left\{-\left[2 \tau / T_{2}(x, y)\right]^{2}\right\}
\end{aligned}
$$

with $1 / T_{2}(x, y)=\Gamma_{\text {non }}+\delta B(x, y) \gamma_{\text {eff }} / 2 \pi$, and the nonmagnetic coherence time $1 / \Gamma_{\text {non }} \sim 0.2 \mathrm{~s}$ being estimated from measurements around the CT (see Sec. VII). Despite the decay of each pixel being Gaussian, we find that the resulting sum decays closer to an exponential, in agreement with the measurements [Fig. 4(a)]. We then fit this exponential and extract the model-predicted $T_{2}\left(f_{\Delta}\right)$. We note that, according to this model, the measured $T_{2}\left(f_{\Delta}\right)$ contains a contribution of vastly different depth-dependent spin-echo decay curves since strain shift $f_{\Delta}$ provides limited resolution in $y$ [Fig. 5(a)].

In order to compare the model to the data, the modelpredicted $T_{2}\left(f_{\Delta}\right)$ can be converted into an effective magnetic noise $\delta B\left(f_{\Delta}\right)=2 \pi /\left[\gamma_{\mathrm{eff}} T_{2}\left(f_{\Delta}\right)\right]$. Figure 5(c) and 5(d) show the experimental and theoretical $\delta B$ as a function of the average position $\langle x\rangle,\langle y\rangle$ of the donors for the three resonators. A semiquantitative agreement for all resonators is obtained for $\sigma_{1}=4 \times 10^{12} \mathrm{~cm}^{-2}$ and $\sigma_{2}=10^{12} \mathrm{~cm}^{-2}$ [see Figs. 5(b)-5(d)]. The numerical calculations, in particular, capture the depth dependence of $T_{2}^{-1}$ for spins. That the shortest $T_{2}$ is for spins close to the wire edge is expected because choosing $B_{0}$ with large strain probes spins very close to both the surface and wire edge as shown in Fig. 2(f). For Res1, the dashed curve in Fig. 5(c) shows the result of the calculation assuming a constant density $\sigma_{1}=\sigma_{2}$; we see that the longer $T_{2}$ time measured below the $5-\mu \mathrm{m}$ wire cannot be reproduced in this way. Defect densities of about $10^{12} \mathrm{~cm}^{-2}$ are consistent with typical defect densities at the $\mathrm{Si} / \mathrm{SiO} 2$ interface [45], and with the numbers reported in Ref. [15]. These numbers are also comparable to the ones inferred from flux-noise measurements in superconducting circuits (in the range $\left.1-5 \times 10^{13} \mathrm{~cm}^{-2}\right)[21,46,47]$.

In all of the above discussion, we disregarded the magnetic response of the superconducting aluminum wire, which may have an impact on the field $\delta B$ generated by the layer of surface dipoles due to the Meissner screening of the magnetic field. This assumption was confirmed by calculations described in Appendix E, which show that the impact of the Meissner screening is negligible in our geometry and cannot explain the spatial dependence of $T_{2}$.

It is interesting to compare our measurements (Fig. 4) to previous decoherence studies of near-surface spins in isotopically purified silicon. Antimony ions of concentration $10^{16} \mathrm{~cm}^{-3}$ implanted at an average depth of $100 \mathrm{~nm}$ in samples without metal electrodes and measured using a bulk EPR spectrometer showed $T_{2}=1 \mathrm{~ms}$ [14]. This value is quite similar to the $3 \mathrm{~ms}$ measured far from the wire, supporting a similar decoherence mechanism in both cases. In a different experiment, individual phosphorus donors [5] were implanted at much lower depths (15-20 nm) and measured in devices with close spacing of metal electrodes. Using the $1 / d^{2}$ scaling of the noise standard deviation, we conclude that such a phosphorus donor in our device would have a coherence time around $100 \mu \mathrm{s}$ at most, whereas 
$T_{2}=1 \mathrm{~ms}$ was reported previously [5]. This difference can be attributed to a better interface quality and/or to the larger value of $B_{0}$ (exceeding 1 Tesla), which leads to a complete polarization of impurity spins at $10 \mathrm{mK}$, contrary to our measurements done at low fields. Deconvolving the two effects would be interesting and could be the subject of future work.

The observation that the surface spin density is reduced below the aluminum wire compared to its value away from the wire may be an indication that aluminum has a passivating effect on dangling bonds at the silicon/silicon-oxide interface. In this context, it is interesting to note that aluminum deposition has been shown to affect the underlying silicon-oxide layer, leading to a reduction of its thickness at the expense of the growth of an aluminumoxide layer $[48,49]$. More work is needed to confirm this observation and understand its physicochemical origin.

\section{DECOHERENCE DUE TO NONMAGNETIC NOISE}

We study the sensitivity of spins to nonmagnetic noise sources by suppressing the dominant magnetic noise contribution near the CT in Res1. As expected from Fig. 4(c), $T_{2}$ increases with decreasing $\gamma_{\text {eff }}$ and reaches a maximum value of $0.3 \mathrm{~s}$ when $\gamma_{\text {eff }}=0$ [Fig. 6(a)]. To examine if $T_{2}$ is influenced by second-order magnetic fluctuations, we estimate its upper bound using $1 / T_{2}=\Gamma_{\text {non }}+$ $\left(k_{1} / 2 \pi\right)\left(d \omega / d B_{0}\right)+\left(k_{1} / 2 \pi\right)^{2}\left(d^{2} \omega / d^{2} B_{0}\right)$, where $\Gamma_{\text {non }}$ describes the decoherence from nonmagnetic origin and $k_{1}$ is a position-dependent average magnetic noise fluctuation. With $k_{1} \sim 5 \mathrm{nT}$ for spins below the inductor [Fig. 4(b)], we obtain $\left(k_{1} / 2 \pi\right)^{2}\left(d^{2} \omega / d^{2} B_{0}\right)=2.5 \times 10^{-6} \mathrm{~s}^{-1}$, many orders of magnitude lower than the measured $1 / T_{2}=3 \mathrm{~s}^{-1}$. Therefore, the magnetic noise evidenced in Sec. VI does not cause the decoherence measured at the CT.

Even though $\gamma_{\text {eff }}=0$ at the CT, flip-flops with neighboring spins are still possible since the transverse spin matrix element $\left\langle 0\left|S_{x}\right| 1\right\rangle=0.25$ is nonzero. This decoherence mechanism (called direct flip-flop [9]) is thus not suppressed at the CT. It was found to be dominant in measurements on bulk-doped bismuth donors in silicon [10], with an observed scaling $T_{2} \simeq 10^{3} \mathrm{~s} \cdot \mu \mathrm{m}^{-3} / \rho$, where $\rho$ is the implanted donor concentration expressed in $\mu \mathrm{m}^{-3}$. In our device, this process would limit $T_{2}$ to about $25 \mathrm{~ms}$ for our $\rho \sim 4 \times 10^{4} \mu \mathrm{m}^{-3}$; therefore, it appears to be strongly suppressed, likely because of the locally inhomogeneous strain shifts already evidenced in Sec. V. Moreover, dynamical decoupling measurements at the CT (see Sec. VIII) are found to extend the coherence time, which is incompatible with a direct flip-flop mechanism. Overall, we thus conclude that decoherence by a direct flipflop mechanism is negligible in our measurements, even at the CT. It is also interesting to note that strain effectively protects the donors from decoherence, as it was also shown to increase the spin-lattice relaxation time [17].
Because the CT happens at a fixed value of $B_{0}$, it is not possible to study the dependence of coherence time on $f_{\Delta}$ as thoroughly as was done for magnetic noise (see Sec. VI). Nevertheless, partial data were obtained in two different ways. First, because of the field-sweep-induced vortices in the superconducting aluminum and variations from one experimental run to another, the resonator frequency $\omega_{0}$ at the CT $\left(B_{0}=27 \mathrm{mT}\right)$ was found to vary over time and from run to run, enabling us to measure $T_{2}$ for various values of $f_{\Delta}$ [full circles in Fig. 6(c)]. Another data set is obtained independently, in the course of a single run, using measurements of $T_{2}$ as a function of $B_{0}$ around the CT [see Fig. 6(a)]. Because of the known dependence of both the bismuth unperturbed transition frequency [green solid line in Fig. 6(a)] and the resonator frequency [dashed black curve in Fig. 6(a)] on $B_{0}$, these measurements probe different values of $f_{\Delta}$. Moreover, we can estimate the magnetic noise contribution using the known transition slopes $\gamma_{\text {eff }}\left(B_{0}\right)$ and $k_{1} \sim 5 \mathrm{nT}$. Subtracting this contribution from the inverse measured coherence time, we obtain $\Gamma_{\text {non }}\left(f_{\Delta}\right)$ [open circles in Fig. 6(c)]. Both data sets are consistent with one another and show that $T_{2}$ at the CT decreases for donors located under the wire in more strained areas (i.e., closer to the wire edges). These observations indicate a spatial dependence of the nonmagnetic noise responsible for decoherence at the CT.
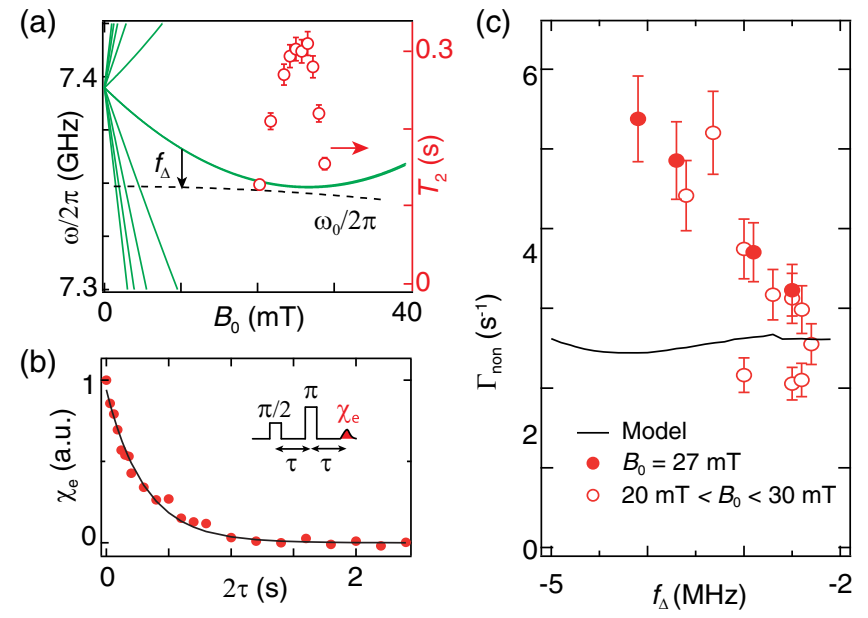

FIG. 6. Nonmagnetic noise characterization at CT in Res1. (a) Calculated spin transition frequencies (green lines), measured resonator frequency (dashed line, left axis), and measured coherence time $T_{2}$ (symbols, right axis) as a function of $B_{0}$. (b) Echo integral $\chi_{e}$ as a function of the delay $2 \tau$ between the $\pi / 2$ pulse and the echo. Red dots are data, and the solid line is an exponential fit yielding a coherence time $T_{2}=300 \mathrm{~ms}$. Magnitude detection is used to circumvent phase noise from the measurement setup. (c) Echo decay rate $\Gamma_{\text {non }}$ as a function of $f_{\Delta}$. Red dots are measurements at $B_{0}=27 \mathrm{mT}$ performed over several runs; red circles are extracted from the data in panel (a). The black solid line is the result of the model described in the main text. 
A likely phenomenon to explain our data is charge noise originating from the interfaces between silicon, silicon oxide, and aluminum oxide, which can produce Stark shifts of the hyperfine constant. Note that $1 / f$ charge noise is ubiquitous in silicon nanoelectronic devices. Its study was revived recently at low temperatures in the context of research on quantum-dot spin qubits [50,51].

As explained in Sec. II, charge noise couples to the $\mathrm{Bi}$ donor spin because of the quadratic Stark effect $\Delta A(\mathbf{E}) /$ $A_{0}=\eta E^{2}$, with $\eta=(-0.26 \pm 0.05) \times 10^{-3} \mu \mathrm{m}^{2} / \mathrm{V}^{2}$. In our device, the donors are subject to a residual electric field of order $E_{\mathrm{r}} \sim 0.1 \mathrm{mV} / \mathrm{nm}$ due to the bulk boron doping. We can therefore estimate the coherence time caused by electric-field fluctuations due to charge noise around the residual field $E_{\mathrm{r}}$ as $T_{2}=2 \pi /\left(10 \eta A_{0} E_{\mathrm{r}} \sqrt{S_{E}}\right)$, assuming fluctuations with a $1 / f$ spectrum $S_{E}(\omega)=S_{E} / \omega$, where $S_{E}$ is the electric-field noise power spectral density at $1 \mathrm{~Hz}$. Using electrostatic simulations, we can, moreover, express the electric-field fluctuations $\sqrt{S_{E}}$ as if they were entirely caused by voltage fluctuations $\sqrt{S_{V}}$ of the aluminum gate electrode. We find that for our device, $\sqrt{S_{V}}=1.5 \mathrm{mV}$ yields a coherence time similar to our measurements [see solid line in Fig. 6(c)]. This level of noise is significantly larger than the gate voltage noise measured in quantum-dot devices $\sqrt{S_{V}} \sim 0.01 \mathrm{mV}[50,51]$, which may indicate that $E_{\mathrm{r}}$ is larger than estimated or that our electrostatic modeling is too crude. We stress that our simplistic reasoning here is only meant to show that typical charge noise levels may indeed limit the coherence time to the values that we measure at the clock transition.

\section{ADDITIONAL NOISE CHARACTERIZATION}

In this section, we present complementary measurements to further characterize the noise responsible for spin decoherence.

First, we use dynamical decoupling to obtain further information about the noise power spectrum $S(\omega)$. Indeed, assuming that $S(\omega)$ scales as $\omega^{-\alpha}$, it can be shown [52-54] that the coherence time measured in a dynamical decoupling sequence containing $N$ refocusing pulses should scale as $N^{\gamma}$, with $\gamma=\alpha /(1+\alpha)$; measuring $\gamma$ therefore gives access to $\alpha$.

We perform coherence time measurements with the Uhrig dynamical decoupling (UDD) and Carr-PurcellMeiboom-Gill (CPMG) sequences. The data are taken with Res1, at $B_{0}=1.4 \mathrm{mT}$ (first transition, giving access to the magnetic component of the noise) and $B_{0}=27 \mathrm{mT}$ (CT, giving access to the charge noise). To obtain reliable data, it was necessary to remove the contribution of spurious echoes due to pulse imperfections using phase cycling [42] (see Appendix D). With both sequences, we find that $T_{2}$ increases with the number $(N)$ of $\pi$ pulses [Fig. 7(a)], with an approximate $N^{1 / 2}$ scaling, both at the first transition and at the CT. This result suggests that the noise power exponent $\alpha$ is close to 1 , indicating an approximate $1 / f$ noise spectrum both for the magnetic noise and the charge noise. We note that a similar $T_{2} \propto$ $N^{1 / 2}$ scaling was observed in $\mathrm{NV}$ centers close to the diamond surface (therefore, probing surface magnetic noise) [55] and in semiconducting quantum dots subjected to charge noise originating from the silicon/silicon-oxide interface [43]. Magnetic noise originating from surface fluctuators is also commonly believed to be responsible for $1 / f$ flux noise in superconducting qubits [56,57]. As discussed earlier, the exponential shape of the echo decay
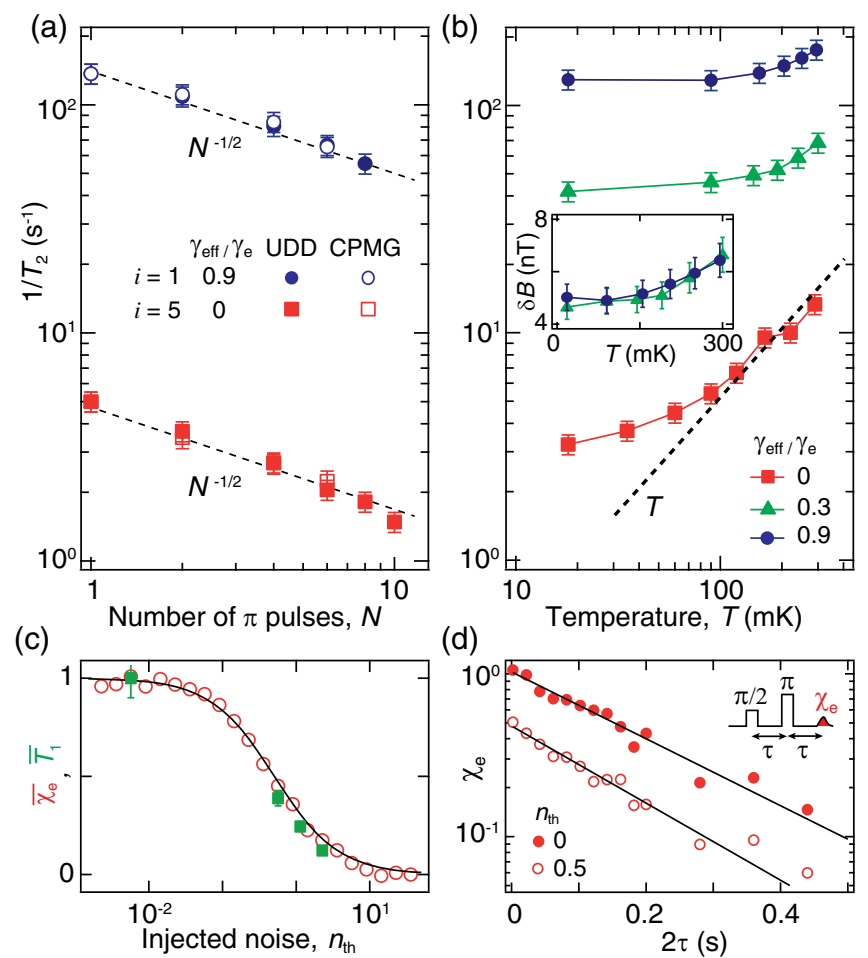

FIG. 7. Additional noise characterizations in Res1 at $f_{\Delta}=$ $-2.5 \mathrm{MHz}$ (spins below the wire). (a) Echo decay rate $1 / T_{2}$ as a function of the number of pulses in the dynamical decoupling sequence on transitions $i=1$ (blue) and $i=5$ (CT, red). Full (open) symbols correspond to the UDD (CPMG) sequence. The dashed line indicates the $N^{-1 / 2}$ scaling. (b) Echo decay rate $1 / T_{2}$ as a function of sample temperature $T$ for transitions $i=1$ (blue), $i=4$ (green), and $i=5$ (CT, red). The dashed line is a guide to the eye, suggesting a $T^{-1}$ scaling at high temperatures of the decay times at the CT. Inset: $\delta B=2 \pi /\left(T_{2} \gamma_{\text {eff }}\right)$ obtained by subtracting the contribution from nonmagnetic noise (values measured at CT), as explained in the main text. (c) Normalized echo amplitude (circles) and spin relaxation time $T_{1}$ (squares) in the presence of injected thermal noise $n_{\text {th }}$. The symbols and line are, respectively, measurements and theory from Eq. (9). (d) Echo integral $\chi_{e}$ as a function of the delay $2 \tau$ at the $i=5$ transition (CT), without (red dots) and with (red circles) injection of $n_{\text {th }}=$ 0.5 photons of thermal noise. Solid lines are exponential fits to the data, and they show that the decay rate is not affected by thermal noise. 
observed on both transitions appears to be the result of an average of Gaussian decays with an inhomogeneous distribution of $T_{2}$ times due to the varying donor depth.

We also measured the coherence time as a function of temperature $T$, at the $\mathrm{CT}\left(T_{2, \mathrm{CT}}\right)$, and the first $\left(T_{2,1 \mathrm{st}}\right)$ and fourth $\left(T_{2,4 \mathrm{th}}\right)$ transitions in Res1. As seen in Fig. 7(b), $T_{2, \mathrm{CT}}(T)$ shows a strong temperature dependence down to the lowest temperatures, whereas $T_{2,1 \mathrm{st}}(T)$ and $T_{2,4 \mathrm{th}}$ have a much weaker temperature dependence. We can estimate the temperature dependence of the purely magnetic noise by computing $T_{2, m}^{-1}(T)=T_{2,1 \mathrm{st} / 4 \mathrm{th}}^{-1}(T)-T_{2, \mathrm{CT}}^{-1}(T)$. Note that this subtraction has no rigorous justification since all measured $T_{2} \mathrm{~s}$ are in fact averages over various Gaussian decays, for which the magnetic and charge decay rates do not add up as simply; nevertheless, $T_{2, m}^{-1}(T)$ has the merit of being directly accessible from the measurements. We see that $T_{2, m}^{-1}(T)$ is unchanged up to $200 \mathrm{mK}$, and it slightly increases up to $300 \mathrm{mK}$. In contrast, $T_{2, \mathrm{CT}}^{-1}(T)$ is 5 times stronger at $300 \mathrm{mK}$ than at $20 \mathrm{mK}$. This different temperature dependence confirms the different physical origin of the noise at the first transition and at the CT. We also note that a strong temperature dependence in the 10-200-mK range was observed for charge noise [51], which further supports our interpretation.

We also quantify the dependence of $T_{2}$ on the presence of thermal photons in the resonator, which are a common source of decoherence for superconducting qubits [58]. To that aim, we inject microwave noise in the input line by connecting the output of a pair of amplifiers in series at room temperature while keeping the sample temperature at $20 \mathrm{mK}$. The average number of thermal noise photons $n_{\text {th }}$ is calibrated using the dependence of both the echo amplitude $\chi_{e}$ and energy relaxation time $T_{1}$ on $n_{\text {th }}$ in the Purcell regime [41],

$$
\frac{\chi_{e}\left(n_{\mathrm{th}}\right)}{\chi_{e}(0)}=\frac{T_{1}\left(n_{\mathrm{th}}\right)}{T_{1}(0)}=\frac{1}{\left(2 n_{\mathrm{th}}+1\right)} .
$$

The data showing $\chi_{e}$ and $T_{1}$ versus $n_{\text {th }}$ are shown in [Fig. 6(c)], closely matching the theoretical prediction and enabling an absolute calibration of $n_{\mathrm{th}}$. We then compare echo decays for $n_{\text {th }}=0$ and 0.5 . For the latter, the thermal noise corresponds to an effective photon temperature of $0.35 \mathrm{~K}$, and both echo amplitude and $T_{1}$ are halved. We extract similar $T_{2}$ values [Fig. 7(d)] and thus conclude that thermal radiation does not limit $T_{2}$ in our device.

\section{CONCLUSION}

We have performed spectral and coherence time studies of shallow-implanted bismuth electron spins in ${ }^{28} \mathrm{Si}$ enriched silicon, using superconducting microresonators with different device geometries and at temperatures between $20 \mathrm{mK}$ and $300 \mathrm{mK}$. We quantitatively describe the spin spectra, which we show to be dominated by strain broadening specific to the sample geometry. Our measurements reveal the existence of locally inhomogeneous strain gradients, which largely suppress both instantaneous diffusion and direct flip-flop decoherence.

The spatially dependent frequency shifts caused by strain enable a spatially resolved measurement of donor spin decoherence. Together with the transition dependence of the effective gyromagnetic ratio, this makes it possible to pinpoint spectral diffusion due to surface paramagnetic impurities as the dominant decoherence mechanism. Our data enable some amount of spatial resolution of the surface spin density, which we estimate to be $10^{12} \mathrm{~cm}^{-2}$ below the aluminum wire and $4 \times 10^{12} \mathrm{~cm}^{-2}$ away from the wire. These figures are comparable to typical dangling bond densities at the silicon/silicon-oxide interface. The physicochemical origin of the reduced defect spin density below the aluminum is unclear. This result, which deserves to be confirmed by further work, is also relevant for the understanding and mitigation of flux noise in superconducting quantum circuits [21]. At the clock transition, the donors are no longer sensitive to magnetic noise. Our measurements suggest that the residual decoherence is caused by charge noise at the sample surface, still enabling us to reach long $T_{2}$ values of up to $300 \mathrm{~ms}$, which confirms that donors in silicon can be used for quantum devices despite the detrimental effect of interfaces [13].

The ability to spatially map noise from interfaces, and, in particular, to clearly distinguish noise below and away from a metallic electrode, is a promising new tool for improving the interface quality and therefore increasing the performance of various quantum devices that use a silicon substrate, including superconducting qubits. Surface passivation methods for improving the quality of superconducting circuits fabricated on silicon (including hydrofluoric acid, forming gas anneal, and deposition of various polymers such as HMDS or self-assembled monolayers [59]) have been explored-the method present here could provide a key to quantitatively understand the impact of these treatments on the surface magnetic and electric noise.

Going further, we note that the use of strain-induced frequency shifts as a tool for spatial mapping of decoherence is by no means restricted to bismuth donors in silicon. Indeed, many, if not all, spin defects in solids are sensitive to strain via some parameter of the spin Hamiltonian: zerofield splitting, hyperfine coupling, nuclear quadrupole, or the g-tensor (for example, by a spin-orbit coupling). As a result, we anticipate the methods demonstrated here could be applied to systems such as acceptors in silicon [18], NV [19] and $\mathrm{SiV}$ [60] centers in diamond, divacancies in $\mathrm{SiC}$ [61], and in rare-earth-doped crystals [20]. Finally, while the use of metallic electrodes with different thermal expansion coefficients to the substrate provides a simple and convenient way to introduce strain, future developments could explore piezoelectric materials to impart a controllable strain gradient across the device and extend the spatial mapping capabilities. 


\section{ACKNOWLEDGMENTS}

We acknowledge technical support from P. Sénat, P.-F. Orfila, and S. Delprat, and are grateful for fruitful discussions within the Quantronics group. We acknowledge IARPA and Lincoln Labs for providing a JTWPA used in the measurements. We acknowledge support from the Horizon 2020 Research and Innovation Program through Grant Agreement No. 771493 (LOQO-MOTIONS), the European Union through the Marie 365 Skłodowska Curie Grant Agreement No. 765267 (QuSCO), and the Agence Nationale de la Recherche (ANR) through projects QIPSE, MIRESPIN (ANR 19 CE47 0011), and the Chaire Industrielle NASNIQ under Contract No. ANR-17-CHIN0001 co-funded by Atos. T. S. was supported by the U.S. Department of Energy under Contract No. DE-AC0205CH11231. J. J. P. was supported by an Australian Research Council Discovery Early Career Research Award (DE190101397).

\section{APPENDIX A: TRANSITION MATRIX ELEMENTS}

A strong electronic-nuclear hyperfine interaction in Si:Bi leads to $18 S_{x}$ transitions at low magnetic fields. In Fig. 1(b), we have labeled those as 1 and 10 for two nondegenerate, and 2 to 9 for eight quasi doubly degenerate transitions. The latter can be recognized in the coupled basis as $|4, m-1\rangle \leftrightarrow|5, m\rangle$ and $|4, m\rangle \leftrightarrow|5, m-1\rangle$, $|m| \leq 4$. Interestingly, two quasidegenerate transitions are complementary, i.e., sum of their $S_{x}$ matrix elements is approximately 0.5 . For example, the clock transition near $27 \mathrm{mT}$ has $S_{x}$ matrix elements of $\left\langle 4,0\left|S_{x}\right| 5,-1\right\rangle=$ $\left\langle 4,-1\left|S_{x}\right| 5,0\right\rangle=0.25$. In contrast, for nondegenerate transitions $|4,-4\rangle \leftrightarrow|5,-5\rangle$ and $|4,4\rangle \leftrightarrow|5,5\rangle$, labeled 1 and 10, respectively, in Fig. 1(b), it is 0.48 . Because matrix elements directly affect spin-photon coupling strength $g_{0}$, Rabi flops are transition dependent [Fig. 8(a)]. The same happens for spin-energy relaxation times as our spins are in (a)

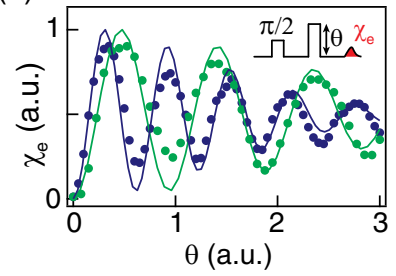

(b)

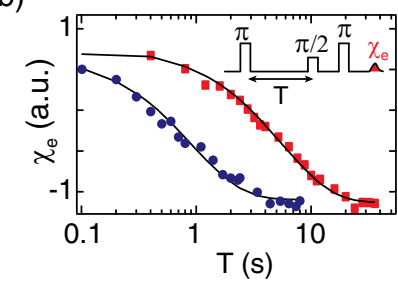

FIG. 8. (a) Measured (symbols) and numerically simulated (lines) Rabi oscillations at transitions $i=1$ (blue) and $i=4$ (green) with Res1. (b) Measured spin-energy relaxation with inversion recovery sequence for the transitions $i=1$ (circles) and CT (squares). Lines are best fits yielding $T_{1}$ of $1 \pm 0.2 \mathrm{~s}$ and $5.5 \pm 1 \mathrm{~s}$, respectively. The resonator loss rate $\kappa$ at CT is $30 \%$ larger than the first transition due to magnetic-field-induced vortices. the strong Purcell limit [34]. The $T_{1}$ measured using inversion recovery sequence for the first transition and CT are shown in Fig. 8 and found to be in excellent agreement with the expected Purcell relaxation time $T_{1}=\kappa / 4 g_{0}^{2}$.

The echo amplitude response across different transitions is complex because of $g_{0}$ variations, which also affect the Rabi angle for a given pulse amplitude and polarization for a given repetition time of the experiment [see Eq. (4)]. For example, Rabi flops performed at $t_{\text {rep }}=4 \mathrm{~s}$ on the fourth transition [Fig. 8(a)] receive dominant echo contribution from $|4,-1\rangle \leftrightarrow|5,-2\rangle$ with a higher matrix element of 0.32 (extrapolated $T_{1}=2.2 \mathrm{~s}$ ) and less so from $|4,-2\rangle \leftrightarrow|5,-1\rangle$ with a matrix element of 0.18 (extrapolated $T_{1}=7 \mathrm{~s}$ ). Such transitiondependent changes in $g_{0}$ are accounted for in the numerical simulations.

\section{APPENDIX B: STRAIN SPECTROSCOPY SIMULATION}

We have performed finite element simulations of strain using COMSOL software. To this end, we input the temperature-dependent thermal expansion coefficients of the materials and their stiffness tensor coefficients [26]. Other input parameters are the thickness of $\mathrm{Al}(50 \pm 5 \mathrm{~nm}$ measured by AFM) and the effective temperature $T_{\text {eff }}$ of the Al evaporation, which we expect to be close to the room temperature at which the sample was fabricated. Both $\mathrm{Al}$ and $\mathrm{Si}$ lattices have cubic symmetry, and therefore, their stiffness tensor $C$ can be simplified as follows:

$$
C=\left(\begin{array}{cccccc}
C_{11} & C_{12} & C_{12} & 0 & 0 & 0 \\
C_{12} & C_{11} & C_{12} & 0 & 0 & 0 \\
C_{12} & C_{12} & C_{11} & 0 & 0 & 0 \\
0 & 0 & 0 & C_{44} & 0 & 0 \\
0 & 0 & 0 & 0 & C_{44} & 0 \\
0 & 0 & 0 & 0 & 0 & C_{44}
\end{array}\right),
$$

with $C_{11}=166 \mathrm{GPa}, C_{12}=64 \mathrm{GPa}$, and $C_{44}=80 \mathrm{GPa}$ for anisotropic $\mathrm{Si}$. However, $\mathrm{Al}$ is modeled as an isotropic material, and its stiffness can be characterized by a Young modulus of $70 \mathrm{GPa}$ and a Poisson ratio of 0.33. Material parameters are summarized in Table II.

TABLE II. Material properties.

\begin{tabular}{lcc}
\hline \hline & Aluminum & Silicon \\
\hline Thermal expansion coefficient $(1 / \mathrm{K})$ & $14.3 \times 10^{-6}$ & $0.7 \times 10^{-6}$ \\
Stiffness tensor $C_{11}, C_{12}, C_{44}(\mathrm{GPa})$ & $103,51,26$ & $166,64,80$ \\
\hline \hline
\end{tabular}




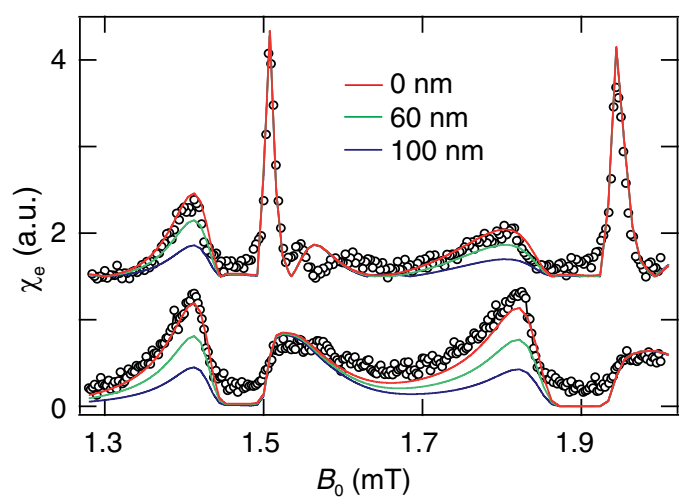

FIG. 9. Simulated spin spectrum (lines) for Res1 when incorporating Schottky barriers of height $0 \mathrm{eV}(0 \mathrm{~nm}), 0.3 \mathrm{eV}(60 \mathrm{~nm})$, and $0.7 \mathrm{eV}(100 \mathrm{~nm})$. Measured data (symbols) at two pulse amplitudes are the same as shown in Fig. 2(a).

\section{APPENDIX C: SCHOTTKY BARRIER}

In this paragraph, we discuss the characteristics of the aluminum/silicon interface and, more precisely, the potential formation of a Schottky barrier. From electrostatic calculation using the known work function of both silicon and aluminum, we would expect the formation of a Schottky barrier, with a depletion zone of depth about $100 \mathrm{~nm}$ for the donor density in our sample. Therefore, a significant fraction of the donors would be ionized, particularly those that are closest to the wire edge, which would have a clear spectroscopic signature. On the other hand, Schottky barriers are notoriously difficult to predict, as the presence of additional charged impurities at the silicon/silicon-oxide/aluminum interface can modify the barrier properties.

We thus use our spectroscopic data as a way to test three Schottky barrier scenarios: a depletion depth of $100 \mathrm{~nm}$ (0.7 eV barrier height), $60 \mathrm{~nm}(0.3 \mathrm{eV})$, and no depletion zone at all. The computed spectra with depletion zones are shown in Fig. 9 with the same measured experimental data as in Fig. 2(a). The comparison shows better agreement for the scenario without any Schottky barrier, which is the only one quantitatively reproducing the relative height of the low- and high-field peaks. Indeed, the shape and dynamics of the Schottky barrier may be significantly affected by the fact that the aluminium strip and silicon are not connected through a circuit to a common reference of chemical potential. The only way $\mathrm{Al}$ and $\mathrm{Si}$ can exchange charges is through the $\mathrm{SiO}_{2}$ oxide, which can be very slow.

\section{APPENDIX D: DYNAMICAL DECOUPLING}

Here, we provide more details on the two dynamical decoupling pulse sequences used in Sec. VIII.

The CPMG sequence consists of a first $\pi / 2_{x}$ pulse, followed, after a duration $\tau / N$, by a sequence of $N \pi_{y}$ pulses separated by $2 \tau / N$, generating an echo at time $2 \tau$ as seen in Fig. 10. In the UDD sequence, the $\pi$ pulses are

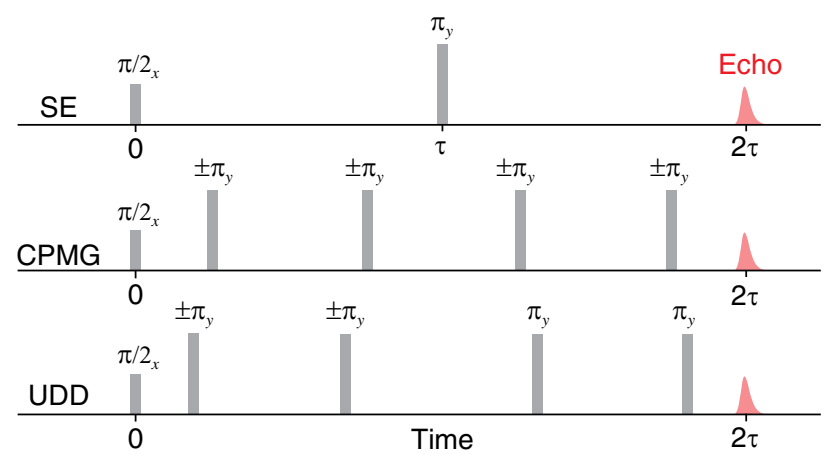

FIG. 10. Pulse sequences for spin echo, CPMG, and UDD $(N=4)$. Phase cycling is done by controlling the sign of the $\pi$ pulses. Subscripts $x, y$ denote the axis of the pulses.

shifted in time compared to the CPMG and occur instead at $2 \tau \sin ^{2}[k \pi /(2 N+2)]$.

In our experiments, the Rabi rotations are not very precise because of the spatial inhomogeneity of the $B_{1}$ field. This feature gives rise to spurious echoes because of the phenomenon of stimulated echoes where two consecutive $\pi / 2$ pulses store coherence as a polarization comb in the frequency domain, which is restored along $S_{x}$ by a third $\pi / 2$ pulse. Such stimulated echoes have a different dependence on the drive pulse phases than the desired echo, resulting from the periodic refocusing of all control pulses. Therefore, phase cycling is the method of choice to measure only the desired echo.

In the CPMG sequence, because of the equidistance of the $\pi$ pulses, many pulse combinations give rise to a stimulated echo at time $2 \tau$. Therefore, a complex phase cycling scheme must be used, varying the phase of each $\pi$ pulse in the sequence (i.e., $2^{N}$ measurements). The UDD sequence is more favorable since most stimulated echoes do not happen at $2 \tau$ and therefore have no overlap with the desired signal. Therefore, only two phase cycling sequences are needed for UDD (all $\pi$ pulses in the first half of the sequence simultaneously take plus or minus sign). The pulse sequences used in this work are shown schematically in Fig. 10.

\section{APPENDIX E: MEISSNER SCREENING}

In order to assess the impact of the Meissner effect on magnetic noise, we have computed the screened magnetic field created by a dipole $|\vec{m}|=\mu_{\mathrm{B}}$ at the $\mathrm{Si} / \mathrm{SiO}_{2}$ interface. We assume a 50-nm-thick type-I superconducting film $(w \rightarrow \infty)$, separated from a Si substrate by a 5 -nm-thick oxide. The screened magnetic field is calculated for a given London penetration depth $\lambda$ as a Fourier-Bessel series solution of Maxwell's equations [62]. The squared magnetic-field component along $z$, which drives spin decoherence [15], is then averaged over all dipole orientations and over a homogeneous distribution of paramagnetic centers at the $\mathrm{Si} / \mathrm{SiO}_{2}$ interface with a density of $\sigma=10^{12} \mathrm{~cm}^{-2}$. In the absence of Meissner screening 


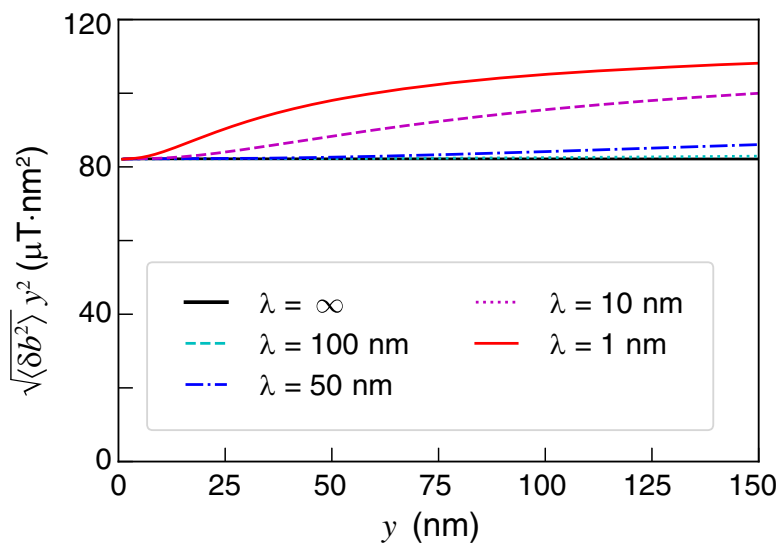

FIG. 11. Calculated Meissner screening of magnetic noise due to the superconducting aluminum for different London penetration depths $\lambda$. The magnetic-field standard deviations along the $z$ axis, $\sqrt{\left\langle\delta b^{2}\right\rangle}$, are plotted for randomly oriented surface magnetic impurities with a uniform density of $\sigma=10^{12} \mathrm{~cm}^{-2}$ and individual magnetic moment of $\mu_{\mathrm{B}}$.

$(\lambda \rightarrow \infty)$, the resulting $\sqrt{\left\langle\delta b^{2}\right\rangle}$ is expected to decrease as $1 / y^{2}$ in the silicon substrate, with $y$ the distance to the $\mathrm{Si} / \mathrm{SiO}_{2}$ interface [15]. Therefore, $\sqrt{\left\langle\delta b^{2}\right\rangle} \times y^{2}$ is plotted for different $\lambda$ 's in Fig. 11. The currents induced in the superconductor screen the dipoles within that material; they also screen perpendicular dipoles within the semiconductor $(\vec{m} \| \vec{y})$ but enhance parallel dipoles $(\vec{m} \| \vec{x}, \vec{z})$. On average, over dipole orientations, Meissner screening results in a net enhancement of $\sqrt{\left\langle\delta b^{2}\right\rangle}$ in the semiconductor. For London penetration depths $\lambda>50 \mathrm{~nm}$, relevant for aluminium, this enhancement remains moderate and cannot explain the experimental data (it would, in fact, reduce coherence times under the $\mathrm{Al}$ strip). Therefore, Meissner screening is not a leading mechanism in the present experiments.

[1] B. E. Kane, A Silicon-Based Nuclear Spin Quantum Computer, Nature (London) 393, 133 (1998).

[2] A. Morello, J. J. Pla, F. A. Zwanenburg, K. W. Chan, K. Y. Tan, H. Huebl, M. Möttönen, C. D. Nugroho, C. Yang, J. A. van Donkelaar, A. D. C. Alves, D. N. Jamieson, C. C. Escott, L. C. L. Hollenberg, R. G. Clark, and A. S. Dzurak, Single-Shot Readout of an Electron Spin in Silicon, Nature (London) 467, 687 (2010).

[3] J. J. Pla, K. Y. Tan, J. P. Dehollain, W. H. Lim, J. J. L. Morton, D. N. Jamieson, A. S. Dzurak, and A. Morello, A Single-Atom Electron Spin Qubit in Silicon, Nature (London) 489, 541 (2012).

[4] M. Veldhorst, J. C. C. Hwang, C. H. Yang, A. W. Leenstra, B. de Ronde, J. P. Dehollain, J. T. Muhonen, F. E. Hudson, K. M. Itoh, A. Morello, and A. S. Dzurak, An Addressable Quantum Dot Qubit with Fault-Tolerant Control-Fidelity, Nat. Nanotechnol. 9, 981 (2014).

[5] J. T. Muhonen, J. P. Dehollain, A. Laucht, F. E. Hudson, R. Kalra, T. Sekiguchi, K. M. Itoh, D. N. Jamieson,
J. C. McCallum, A. S. Dzurak, and A. Morello, Storing Quantum Information for 30 Seconds in a Nanoelectronic Device, Nat. Nanotechnol. 9, 986 (2014).

[6] C. H. Yang, R. C. C. Leon, J. C. C. Hwang, A. Saraiva, T. Tanttu, W. Huang, J. C. Lemyre, K. W. Chan, K. Y. Tan, F. E. Hudson, K. M. Itoh, A. Morello, M. Pioro-Ladrière, A. Laucht, and A. S. Dzurak, Operation of a Silicon Quantum Processor Unit Cell above One Kelvin, Nature (London) 580, 350 (2020).

[7] L. Petit, H. G. J. Eenink, M. Russ, W. I. L. Lawrie, N. W. Hendrickx, S. G. J. Philips, J. S. Clarke, L. M. K. Vandersypen, and M. Veldhorst, Universal Quantum Logic in Hot Silicon Qubits, Nature (London) 580, 355 (2020).

[8] A. Morello, J. J. Pla, P. Bertet, and D. N. Jamieson, Donor Spins in Silicon for Quantum Technologies, Adv. Quantum Technol. 3, 2000005 (2020).

[9] A. M. Tyryshkin, S. Tojo, J. J. L. Morton, H. Riemann, N. V. Abrosimov, P. Becker, H.-J. Pohl, T. Schenkel, M. L. W. Thewalt, K. M. Itoh, and S. A. Lyon, Electron Spin Coherence exceeding Seconds in High-Purity Silicon, Nat. Mater. 11, 143 (2012).

[10] G. Wolfowicz, A. M. Tyryshkin, R. E. George, H. Riemann, N. V. Abrosimov, P. Becker, H.-J. Pohl, M. L. W. Thewalt, S. A. Lyon, and J. J. L. Morton, Atomic Clock Transitions in Silicon-Based Spin Qubits, Nat. Nanotechnol. 8, 561 (2013).

[11] B. Julsgaard, C. Grezes, P. Bertet, and K. Molmer, Quantum Memory for Microwave Photons in an Inhomogeneously Broadened Spin Ensemble, Phys. Rev. Lett. 110, 250503 (2013).

[12] J. J. L. Morton and P. Bertet, Storing Quantum Information in Spins and High-Sensitivity ESR, J. Magn. Reson. 287, 128 (2018).

[13] V. Ranjan, J. O'Sullivan, E. Albertinale, B. Albanese, T. Chanelière, T. Schenkel, D. Vion, D. Esteve, E. Flurin, J. J. L. Morton, and P. Bertet, Multimode Storage of Quantum Microwave Fields in Electron Spins over 100 ms, Phys. Rev. Lett. 125, 210505 (2020).

[14] T. Schenkel, J. A. Liddle, A. Persaud, A. M. Tyryshkin, S. A. Lyon, R. de Sousa, K. B. Whaley, J. Bokor, J. Shangkuan, and I. Chakarov, Electrical Activation and Electron Spin Coherence of Ultralow Dose Antimony Implants in Silicon, Appl. Phys. Lett. 88, 112101 (2006).

[15] R. de Sousa, Dangling-Bond Spin Relaxation and Magnetic $1 / f$ Noise from the Amorphous-Semiconductor/Oxide Interface: Theory, Phys. Rev. B 76, 245306 (2007).

[16] S.-Y. Paik, S.-Y. Lee, W. J. Baker, D. R. McCamey, and C. Boehme, $T_{1}$ and $T_{2}$ Spin Relaxation Time Limitations of Phosphorous Donor Electrons Near Crystalline Silicon to Silicon Dioxide Interface Defects, Phys. Rev. B 81, 075214 (2010).

[17] S. B. Tenberg, S. Asaad, M. T. Mądzik, M. A. I. Johnson, B. Joecker, A. Laucht, F. E. Hudson, K. M. Itoh, A. M. Jakob, B. C. Johnson, D. N. Jamieson, J. C. McCallum, A.S. Dzurak, R. Joynt, and A. Morello, Electron Spin Relaxation of Single Phosphorus Donors in Metal-OxideSemiconductor Nanoscale Devices, Phys. Rev. B 99, 205306 (2019).

[18] T. Kobayashi, J. Salfi, C. Chua, J. van der Heijden, M. G. House, D. Culcer, W. D. Hutchison, B. C. Johnson, 
J. C. McCallum, H. Riemann, N. V. Abrosimov, P. Becker, H.-J. Pohl, M. Y. Simmons, and S. Rogge, Engineering Long Spin Coherence Times of Spin-Orbit Qubits in Silicon, Nat. Mater. 20, 38 (2021).

[19] P. Udvarhelyi, V. O. Shkolnikov, A. Gali, G. Burkard, and A. Pályi, Spin-Strain Interaction in Nitrogen-Vacancy Centers in Diamond, Phys. Rev. B 98, 075201 (2018).

[20] Q. Zhang, G. Hu, G. G. de Boo, M. Rančić, B. C. Johnson, J. C. McCallum, J. Du, M. J. Sellars, C. Yin, and S. Rogge, Single Rare-Earth Ions as Atomic-Scale Probes in Ultrascaled Transistors, Nano Lett. 19, 5025 (2019).

[21] J. Braumüller, L. Ding, A. P. Vepsäläinen, Y. Sung, M. Kjaergaard, T. Menke, R. Winik, D. Kim, B. M. Niedzielski, A. Melville, J. L. Yoder, C. F. Hirjibehedin, T. P. Orlando, S. Gustavsson, and W. D. Oliver, Characterizing and Optimizing Qubit Coherence Based on SQUID Geometry, Phys. Rev. Applied 13, 054079 (2020).

[22] J. Mansir, P. Conti, Z. Zeng, J. J. Pla, P. Bertet, M. W. Swift, C. G. Van de Walle, M. L. W. Thewalt, B. Sklenard, Y. M. Niquet, and J. J. L. Morton, Linear Hyperfine Tuning of Donor Spins in Silicon Using Hydrostatic Strain, Phys. Rev. Lett. 120, 167701 (2018).

[23] A. Bienfait, J. J. Pla, Y. Kubo, M. Stern, X. Zhou, C. C. Lo, C. D. Weis, T. Schenkel, M. L. W. Thewalt, D. Vion, D. Esteve, B. Julsgaard, K. Mølmer, J. J. L. Morton, and P. Bertet, Reaching the Quantum Limit of Sensitivity in Electron Spin Resonance, Nat. Nanotechnol. 11, 253 (2016).

[24] S. Probst, A. Bienfait, P. Campagne-Ibarcq, J. J. Pla, B. Albanese, J. F. Da Silva Barbosa, T. Schenkel, D. Vion, D. Esteve, K. Moelmer, J. J. L. Morton, R. Heeres, and P. Bertet, Inductive-Detection Electron-Spin Resonance Spectroscopy with 65 Spins $\left(/ \mathrm{Hz}^{1 / 2}\right)$ Sensitivity, Appl. Phys. Lett. 111, 202604 (2017).

[25] V. Ranjan, S. Probst, B. Albanese, T. Schenkel, D. Vion, D. Esteve, J. J. L. Morton, and P. Bertet, Electron Spin Resonance Spectroscopy with Femtoliter Detection Volume, Appl. Phys. Lett. 116, 184002 (2020).

[26] J. J. Pla, A. Bienfait, G. Pica, J. Mansir, F. A. Mohiyaddin, Z. Zeng, Y. M. Niquet, A. Morello, T. Schenkel, J. J. L. Morton, and P. Bertet, Strain-Induced Spin-Resonance Shifts in Silicon Devices, Phys. Rev. Applied 9, 044014 (2018).

[27] J. O'Sullivan, O. W. Kennedy, C. W. Zollitsch, M. Šimėnas, C. N. Thomas, L. V. Abdurakhimov, S. Withington, and J. J. L. Morton, Spin-Resonance Linewidths of Bismuth Donors in Silicon Coupled to Planar Microresonators, Phys. Rev. Applied 14, 064050 (2020).

[28] G. Feher, Electron Spin Resonance Experiments on Donors in Silicon. I. Electronic Structure of Donors by the Electron Nuclear Double Resonance Technique, Phys. Rev. 114, 1219 (1959).

[29] M. H. Mohammady, G. W. Morley, and T. S. Monteiro, Bismuth Qubits in Silicon: The Role of EPR Cancellation Resonances, Phys. Rev. Lett. 105, 067602 (2010).

[30] G. Pica, G. Wolfowicz, M. Urdampilleta, M. L. W. Thewalt, H. Riemann, N. V. Abrosimov, P. Becker, H.-J. Pohl, J. J. L. Morton, R. N. Bhatt, S. A. Lyon, and B. W. Lovett, Hyperfine Stark Effect of Shallow Donors in Silicon, Phys. Rev. B 90, 195204 (2014).
[31] S. Probst, V. Ranjan, Q. Ansel, R. Heeres, B. Albanese, E. Albertinale, D. Vion, D. Esteve, S. J. Glaser, D. Sugny, and P. Bertet, Shaped Pulses for Transient Compensation in Quantum-Limited Electron Spin Resonance Spectroscopy, J. Magn. Reson. 303, 42 (2019).

[32] T. Van Duzer and C. W. Turner, Principles of Superconductive Devices and Circuits (Prentice-Hall PTR, Englewood Cliffs, NJ, 1999).

[33] E. M. Purcell, Spontaneous Emission Probabilities at Radio Frequencies, Phys. Rev. 69, 681 (1946).

[34] A. Bienfait, J. J. Pla, Y. Kubo, X. Zhou, M. Stern, C.-C. Lo, C. D. Weis, T. Schenkel, D Vion, D. Esteve, J. J. L. Morton, and P. Bertet, Controlling Spin Relaxation with a Cavity, Nature (London) 531, 74 (2016).

[35] C. Eichler, A. J. Sigillito, S. A. Lyon, and J. R. Petta, Electron Spin Resonance at the Level of $10^{4}$ Spins Using Low Impedance Superconducting Resonators, Phys. Rev. Lett. 118, 037701 (2017).

[36] C. Macklin, K. O'Brien, D. Hover, M. E. Schwartz, V. Bolkhovsky, X. Zhang, W. D. Oliver, and I. Siddiqi, A Near-Quantum-Limited Josephson Traveling-Wave Parametric Amplifier, Science 350, 307 (2015).

[37] V. Ranjan, S. Probst, B. Albanese, A. Doll, O. Jacquot, E. Flurin, R. Heeres, D. Vion, D. Esteve, J. J. L. Morton, and P. Bertet, Pulsed Electron Spin Resonance Spectroscopy in the Purcell Regime, J. Magn. Reson. 310, 106662 (2020).

[38] S. Asaad, V. Mourik, B. Joecker, M. A. I. Johnson, A. D. Baczewski, H. R. Firgau, and M. T. Mądzik, V. Schmitt, J. J. Pla, F. E. Hudson, K. M. Itoh, J. C. McCallum, A. S. Dzurak, A. Laucht, and A. Morello, Coherent Electrical Control of a Single High-Spin Nucleus in Silicon, Nature (London) 579, 205 (2020).

[39] C. D. Weis, C. C. Lo, V. Lang, A. M. Tyryshkin, R. E. George, K. M. Yu, J. Bokor, S. A. Lyon, J. J. L. Morton, and T. Schenkel, Electrical Activation and Electron Spin Resonance Measurements of Implanted Bismuth in Isotopically Enriched Silicon-28, Appl. Phys. Lett. 100, 172104 (2012).

[40] T. Sekiguchi, M. Steger, K. Saeedi, M. L. W. Thewalt, H. Riemann, N. V. Abrosimov, and N. Nötzel, Hyperfine Structure and Nuclear Hyperpolarization Observed in the Bound Exciton Luminescence of Bi Donors in Natural Si, Phys. Rev. Lett. 104, 137402 (2010).

[41] B. Albanese, S. Probst, V. Ranjan, C. W. Zollitsch, M. Pechal, A. Wallraff, J. J. L. Morton, D. Vion, D. Esteve, E. Flurin, and P. Bertet, Radiative Cooling of a Spin Ensemble, Nat. Phys. 16, 751 (2020).

[42] A. Schweiger and G. Jeschke, Principles of Pulse Electron Paramagnetic Resonance (Oxford University Press, New York, 2001).

[43] J. Yoneda, K. Takeda, T. Otsuka, T. Nakajima, M. R. Delbecq, G. Allison, T. Honda, T. Kodera, S. Oda, Y. Hoshi, N. Usami, K. M. Itoh, and S. Tarucha, A QuantumDot Spin Qubit with Coherence Limited by Charge Noise and Fidelity Higher than 99.9\%, Nat. Nanotechnol. 13, 102 (2018).

[44] G. Ithier, E. Collin, P. Joyez, P. J Meeson, D. Vion, D. Esteve, F. Chiarello, A. Shnirman, Y. Makhlin, J. Schriefl, and G. Schön, Decoherence in a Superconducting Quantum Bit Circuit, Phys. Rev. B 72, 134519 (2005). 
[45] G. Van Gorp and A. Stesmans, Dipolar Interaction between [111] $P_{b}$ Defects at the (111) $\mathrm{Si} / \mathrm{SiO}_{2}$ Interface Revealed by Electron-Spin Resonance, Phys. Rev. B 45, 4344 (1992).

[46] R. H. Koch, D. P. DiVincenzo, and J. Clarke, Model for $1 / f$ Flux Noise in SQUIDs and Qubits, Phys. Rev. Lett. 98, 267003 (2007).

[47] S. Sendelbach, D. Hover, A. Kittel, M. Mück, J. M. Martinis, and R. McDermott, Magnetism in SQUIDs at Millikelvin Temperatures, Phys. Rev. Lett. 100, 227006 (2008).

[48] W. H. Lim, H. Huebl, L. H. Willems van Beveren, S. Rubanov, P. G. Spizzirri, S. J. Angus, R. G. Clark, and A. S. Dzurak, Electrostatically Defined Few-Electron Double Quantum Dot in Silicon, Appl. Phys. Lett. 94, 173502 (2009).

[49] P. C. Spruijtenburg, S. V. Amitonov, W. G. van der Wiel, and F. A. Zwanenburg, A Fabrication Guide for Planar Silicon Quantum Dot Heterostructures, Nanotechnology 29, 143001 (2018).

[50] L. Petit, J. M. Boter, H. G. J. Eenink, G. Droulers, M. L. V. Tagliaferri, R. Li, D. P. Franke, K. J. Singh, J. S. Clarke, R. N. Schouten, V. V. Dobrovitski, L. M. K. Vandersypen, and M. Veldhorst, Spin Lifetime and Charge Noise in Hot Silicon Quantum Dot Qubits, Phys. Rev. Lett. 121, 076801 (2018).

[51] E. J. Connors, J. J. Nelson, H. Qiao, L. F. Edge, and J. M. Nichol, Low-Frequency Charge Noise in $\mathrm{Si} / \mathrm{SiGe}$ Quantum Dots, Phys. Rev. B 100, 165305 (2019).

[52] G. A. Álvarez and D. Suter, Measuring the Spectrum of Colored Noise by Dynamical Decoupling, Phys. Rev. Lett. 107, 230501 (2011).

[53] T. Yuge, S. Sasaki, and Y. Hirayama, Measurement of the Noise Spectrum Using a Multiple-Pulse Sequence, Phys. Rev. Lett. 107, 170504 (2011).

[54] J. Medford, Ł. Cywiński, C. Barthel, C. M. Marcus, M. P. Hanson, and A. C. Gossard, Scaling of Dynamical Decoupling for Spin Qubits, Phys. Rev. Lett. 108, 086802 (2012).
[55] B. A. Myers, A. Das, M. C. Dartiailh, K. Ohno, D. D. Awschalom, and A.C. B. Jayich, Probing Surface Noise with Depth-Calibrated Spins in Diamond, Phys. Rev. Lett. 113, 027602 (2014).

[56] F. Yoshihara, K. Harrabi, A. O. Niskanen, Y. Nakamura, and J.S. Tsai, Decoherence of Flux Qubits due to $1 / f$ Flux Noise, Phys. Rev. Lett. 97, 167001 (2006).

[57] J. Bylander, S. Gustavsson, F. Yan, F. Yoshihara, K. Harrabi, G. Fitch, D. G. Cory, Y. Nakamura, J.-S. Tsai, and W. D. Oliver, Noise Spectroscopy through Dynamical Decoupling with a Superconducting Flux Qubit, Nat. Phys. 7, 565 (2011).

[58] P. Bertet, I. Chiorescu, G. Burkard, K. Semba, C. J. P. M. Harmans, D. P. DiVincenzo, and J. E. Mooij, Dephasing of a Superconducting Qubit Induced by Photon Noise, Phys. Rev. Lett. 95, 257002 (2005).

[59] A. Bruno, G. de Lange, S. Asaad, K. L. van der Enden, N. K. Langford, and L. DiCarlo, Reducing Intrinsic Loss in Superconducting Resonators by Surface Treatment and Deep Etching of Silicon Substrates, Appl. Phys. Lett. 106, 182601 (2015).

[60] S. Meesala, Y.-I. Sohn, B. Pingault, L. Shao, H. A. Atikian, J. Holzgrafe, M. Gündoğan, C. Stavrakas, A. Sipahigil, C. Chia, R. Evans, M. J. Burek, M. Zhang, L. Wu, J. L. Pacheco, J. Abraham, E. Bielejec, M. D. Lukin, M. Atatüre, and M. Lončar, Strain Engineering of the Silicon-Vacancy Center in Diamond, Phys. Rev. B 97, 205444 (2018).

[61] A. L. Falk, P. V. Klimov, B. B. Buckley, V. Ivády, I. A. Abrikosov, G. Calusine, W. F. Koehl, Á. Gali, and D. D. Awschalom, Electrically and Mechanically Tunable Electron Spins in Silicon Carbide Color Centers, Phys. Rev. Lett. 112, 187601 (2014).

[62] M. V. Milošević, S. V. Yampolskii, and F. M. Peeters, Magnetic Pinning of Vortices in a Superconducting Film: The (Anti)vortex-Magnetic Dipole Interaction Energy in the London Approximation, Phys. Rev. B 66, 174519 (2002). 